\title{
Polycystin-1 interacts with TAZ to stimulate osteoblastogenesis and inhibit adipogenesis
}

\author{
Zhousheng Xiao, ${ }^{1}$ Jerome Baudry, ${ }^{2,3}$ Li Cao, ${ }^{1}$ Jinsong Huang, ${ }^{1}$ Hao Chen, ${ }^{4}$ Charles R. Yates, ${ }^{4}$ Wei Li, ${ }^{4}$ Brittany Dong, ${ }^{5}$ \\ Christopher M. Waters, ${ }^{5}$ Jeremy C. Smith, ${ }^{2,3}$ and L. Darryl Quarles ${ }^{1}$ \\ 'Department of Medicine, University of Tennessee Health Science Center, Memphis, Tennessee, USA. UT/ORNL Center for Molecular Biophysics, Oak Ridge National Laboratory, Oak Ridge, Tennessee, USA. \\ ${ }^{3}$ Department of Biochemistry and Cellular and Molecular Biology, University of Tennessee, Knoxville, Tennessee, USA. ${ }^{4}$ Department of Pharmaceutical Sciences and ${ }^{5}$ Department of Physiology, University of \\ Tennessee Health Science Center, Memphis, Tennessee, USA.
}

\begin{abstract}
The molecular mechanisms that transduce the osteoblast response to physical forces in the bone microenvironment are poorly understood. Here, we used genetic and pharmacological experiments to determine whether the polycystins PC1 and PC2 (encoded by Pkd1 and Pkd2) and the transcriptional coactivator TAZ form a mechanosensing complex in osteoblasts. Compound-heterozygous mice lacking 1 copy of Pkd1 and Taz exhibited additive decrements in bone mass, impaired osteoblast-mediated bone formation, and enhanced bone marrow fat accumulation. Bone marrow stromal cells and osteoblasts derived from these mice showed impaired osteoblastogenesis and enhanced adipogenesis. Increased extracellular matrix stiffness and application of mechanical stretch to multipotent mesenchymal cells stimulated the nuclear translocation of the PC1 C-terminal tail/TAZ (PC1-CTT/TAZ) complex, leading to increased runt-related transcription factor 2-mediated (Runx2-mediated) osteogenic and decreased PPAR $\gamma$-dependent adipogenic gene expression. Using structure-based virtual screening, we identified a compound predicted to bind to PC2 in the PC1:PC2 C-terminal tail region with helix:helix interaction. This molecule stimulated polycystin- and TAZ-dependent osteoblastogenesis and inhibited adipogenesis. Thus, we show that polycystins and TAZ integrate at the molecular level to reciprocally regulate osteoblast and adipocyte differentiation, indicating that the polycystins/TAZ complex may be a potential therapeutic target to increase bone mass.
\end{abstract}

\section{Introduction}

Skeletal loading and unloading have major effects on skeletal homeostasis. In contrast to postmenopausal osteoporosis, in which bone resorption plays a major role in bone loss, and age-related osteoporosis and conditions of skeletal unloading, such as occurs due to microgravity, immobility, and/or sarcopenia, a decrease in osteoblast-mediated bone formation accompanied by a gain of marrow fat underlies the pathogenesis of osteopenia (1-4). On the other hand, mechanical loading from exercise, vibration, or pulsed electromagnetic fields leads to a gain in bone mass due to stimulation of osteoblastogenesis and inhibition of bone marrow adipogenesis (5-7). The molecular identity of the physiologically relevant mechanosensor in bone, and the cellular mechanisms responsible for the reciprocal control of osteoblastogenesis and adipogenesis during skeletal unloading, are uncertain (8-10).

Polycystin-1 (PC1, encoded by the PKD1 gene) and polycystin-2 (PC2, encoded by the PKD2 gene) form a prototypic mechanosensing complex in the kidney and other tissues. PC1, an adhesion-like receptor $(11,12)$, and PC2, a nonselective ion channel (13), interact through their respective coiled-coil domains to form a complex

Authorship note: Z. Xiao and J. Baudry contributed equally to this work. Conflict of interest: The authors have declared that no conflict of interest exists. Submitted: March 2, 2017; Accepted: October 17, 2017.

Reference information: / Clin Invest. 2018;128(1):157-174.

https://doi.org/10.1172/JCI93725. that senses fluid-shear stress in renal epithelial cells (14-17). Inactivating mutations of either $P K D 1$ or $P K D 2$ cause renal cystic disease in mice $(18,19)$ and autosomal dominant polycystic kidney disease (ADPKD) in humans $(20,21)$.

Polycystins are also expressed in other tissues $(22,23)$, and several extrarenal functions are being recognized $(23,24)$, including in the skeleton $(25,26)$, where recent evidence shows that the polycystin complex plays an important role in osteoblastogenesis to control bone formation (27-29). In this regard, osteoblastspecific deletion of $P k d 1$ or $P k d 2$ resulted in osteopenia, reduced runt-related transcription factor 2-dependent (Runx2-dependent) osteoblastogenesis, and impaired bone mechanosensing responses to in vivo mechanical loading in mice (30-32). While loss of $P k d 1$ and $P k d 2$ have concordant effects on osteoblast-mediated bone formation, they exhibit opposite effects on bone marrow adipogenesis (30-32). Indeed, loss of $P k d 1$ increases PPAR $\gamma$-dependent adipogenesis, leading to increased bone marrow fat, while loss of $P k d 2$ results in decreased adipogenesis and suppression of both Runx 2 and PPAR $\gamma$. The discordant effects on adipogenesis suggest that PC1 and PC2 functions can be uncoupled and that alternative, PC2-independent pathways link PC1 to adipogenesis (30-32).

TAZ (transcriptional coactivator with a PDZ-binding domain; also known as WW domain-containing transcription regulator 1, or WWTR1), a downstream effector of the Hippo pathway, is a candidate for linking PC1 to adipogenesis. TAZ is regulated by extracellular mechanical stimuli that involve cytoskeletal-dependent nuclear shuffling in response to alterations in extracellular matrix 
stiffness (33-35). There is evidence that PC1 and TAZ may interact to cause polycystic kidney disease through a common pathway. Global Taz knockout and conditional deletion of Taz from the kidney lead to cystic kidney disease in mice $(36,37)$; and in renal epithelial cells, TAZ binds to the PC1 C-terminal tail (PC1-CTT) to facilitate nuclear translocation (38-40), and binds to PC2-CTT to enhance PC2 degradation (41). TAZ also differentially regulates osteoblastogenesis and adipogenesis (42). Nuclear translocation of TAZ coactivates Runx2 to stimulate osteoblastogenesis (43) and represses PPAR $\gamma$ to inhibit adipogenesis (44) in vitro. Moreover, transgenic overexpression of Taz in osteoblasts leads to increased osteoblast-mediated bone formation and decreased bone marrow adipogenesis (45); depletion of taz in zebrafish impairs bone development (42), and $\mathrm{Taz}^{-/-}$mice have small stature and ossification defects (36). These observations raise the possibility that TAZ may be an essential component of the polycystin mechanosensing pathway in bone that regulates bone mass through the reciprocal control of osteoblastogenesis and adipogenesis.

In the current study, we investigated whether TAZ mediates the differential effects of PC1 on osteoblastogenesis and adipogenesis in both in vivo mouse genetic models and in vitro cell culture models. We found that PC1-CTT interacts with TAZ in osteoblasts to integrate 2 distinct mechanosensing pathways, leading to stimulation of Runx2-mediated osteoblastogenesis and inhibition of PPAR $\gamma$-mediated adipogenesis in the skeleton. Furthermore, using 3D ensemble docking algorithms, we identify a small molecule, predicted to bind to PC2 in the PC1:PC2 interacting region, that stimulated osteoblastogenesis and inhibited adipogenesis in vitro and in vivo. Hence, the polycystins/TAZ complex is a potential target for pharmaceutical development of small molecules to mimic mechanical loading to increase bone mass.

\section{Results}

Additive effects of combined Taz and Pkd1 deficiency on bone mass and lineage commitment. We explored genetic interactions between PC1 and TAZ in the skeleton by characterizing the effects of singleand compound-heterozygous loss of $P k d 1$ and Taz on bone mineral density (BMD). This was accomplished by crossing of heterozygous $\mathrm{Taz}^{+/-}$mice with heterozygous $P k d 1^{+/-}$mice to create doubleheterozygous $\mathrm{Taz}^{+/-} ; \mathrm{Pkd1}^{+/-}$animals. These mice were born at the expected Mendelian frequency, and all genotypes had normal survival, gross appearance, and body weight indistinguishable from those of WT mice over the period of study. Neither single- nor compound-mutant mice developed cystic kidney disease by gross and histological inspection of the kidneys (data not shown).

Adult male and female $P k d 1^{+-}$mice exhibited a low BMD by dual-energy x-ray absorptiometry scan analysis. This was due to a reduction in trabecular bone volume $(13.2 \%)$, cortical bone thickness (9.2\%), and impaired osteoblast-mediated mineral apposition rates $(26.6 \%)$, as previously reported in 6-week-old mice (46). In contrast, neither male nor female $\mathrm{Taz}^{+/-}$mice had abnormalities in bone mass, bone structure, and bone formation rate compared with WT mice (Figure 1). However, combined loss of 1 allele of Taz and $P k d 1$ resulted in additive reductions in $\mathrm{BMD}$, as evidenced by the $21 \%$ reduction in BMD in the doubleheterozygous $\mathrm{Taz}^{+/-} ; \mathrm{Pkd1}^{+/-}$mice (Figure 1A). Micro-CT analysis revealed that double-heterozygous $\mathrm{Taz}^{+/-} ; \mathrm{Pkd1}^{+/-}$mice had greater loss in both trabecular (26.3\%) and cortical bone (19.4\%) than did single-heterozygous mice (Figure 1B). These reductions in bone volume were associated with a significant decrease in mineral apposition rates in single-heterozygous $\mathrm{Taz}^{+/-}$and $\mathrm{Pkd1}^{+/-}$mice compared with age-matched WT mice and an even greater reduction (44.6\%) in double-heterozygous $\mathrm{Taz}^{+/} ; \mathrm{Pkd1}^{+/-}$mice (Figure 1C). Finally, bone marrow exhibited an increased percentage of fat cells in single-heterozygous $\mathrm{Pkd1}^{+/-}$mice compared with age-matched WT mice and even greater increments in double-heterozygous $\mathrm{Taz}^{+/-} ; P k d 1^{+/-}$mice at 8 weeks of age, as evidenced by a much higher number of adipocytes and fat droplets in decalcified tibiae stained with osmium tetroxide $\left(\mathrm{OsO}_{4}\right)$ (Figure $\left.1 \mathrm{D}\right)$, indicating an enhancement of adipogenesis in double-heterozygous $\mathrm{Taz}^{+/-} ; \mathrm{Pkd1}^{+/-}$mice. Consistent with low-turnover osteopenia with a reduction in osteoclast activity, histomorphometric analysis of the femurs by tartrateresistant acid phosphatase (TRAP) immunostaining showed reduced osteoclast surface per bone surface in $\mathrm{Pkd1}^{+/-}$and $\mathrm{Taz}^{+/-}$; $\mathrm{Pkd1}^{+/-}$mice compared with WT and $\mathrm{Taz}^{+/-}$mice (Figure 1E).

To investigate whether combined TAZ and $P k d 1$ deficiency resulted in additive effects on gene expression profiles in bone, we examined by real-time reverse transcription PCR (RT-PCR) the expression levels of a panel of osteoblast-, osteoclast-, and chondrocyte-related mRNAs in the femurs of 8-week-old WT, heterozygous $\mathrm{Taz}^{+/}$, heterozygous $\mathrm{Pkd1}^{+/-}$, and double-heterozygous $\mathrm{Taz}^{+/-} ; \mathrm{Pkd1}^{+/-}$mice (Table 1). Single-heterozygous $\mathrm{Taz}^{+/-}$showed normal bone gene expression profiles except $50 \%$ reduction of Taz transcripts (Table 1). However, bone derived from singleheterozygous $\mathrm{Pkd1}^{+/-}$mice had measurable reductions in osteocalcin, osteopontin, osteoprotegerin (Opg), RANK ligand (RankL), $M m p 13$, sclerostin (Sost), and Dmp1 mRNA levels in comparison with WT mice. Significantly greater reductions of osteoblasts and osteocyte-related genes were observed in double-heterozygous $\mathrm{Taz}^{+/-} ; P k d 1^{+/-}$mice for osteocalcin, RankL, Mmp13, Sost, and Dmp1. Bone expression of Trap and Mmp9, markers of bone resorption, were reduced in heterozygous $P k d 1^{+/-}$mice and to a greater extent in double-heterozygous $\mathrm{Taz}^{+/} ; \mathrm{Pkd1}^{+/}$mice compared with WT littermates (Table 1), suggesting that bone loss was mainly due to decreased osteoblast-mediated bone formation. These findings suggest that a low bone formation rate rather than increased bone resorption accounts for the low BMD and bone volume of femurs in single-heterozygous $\mathrm{Pkd1}^{+/-}$and double-heterozygous $\mathrm{Taz}^{+/-} ; \mathrm{Pkd1}^{+/-}$deficient mice. Pparg, an adipocyte transcription factor, and adipocyte markers, including lipoprotein lipase $(L p l)$ and adipocyte fatty acid-binding protein $2(a P 2)$, were significantly increased in femurs of heterozygous $P k d 1^{+/-}$mice and to a greater extent in double-heterozygous $\mathrm{Taz}^{+/-} ; P k d 1^{+/-}$mice compared with WT littermates (Table 1). Transcripts of chondrocyte-related genes did not differ between single- and double-heterozygous $\mathrm{Taz}^{+/-} ; P k d 1^{+/-}$mice (Table 1). Thus, Taz deficiency and Pkd1 deficiency are additive on both osteogenesis and adipogenesis.

Additive effects of combined Taz and Pkd1 deficiency on osteoblast differentiation. To explore the mechanism underlying the increase in osteoblastogenesis and decreased adipogenesis in doubleheterozygous $\mathrm{Taz}^{+-} ; \mathrm{Pkd1}^{+/}$mice, we performed bone marrowderived stromal cell (BMSC) cultures derived from age-matched WT, heterozygous $\mathrm{Taz}^{+-}$, heterozygous $\mathrm{Pkd1}^{+/}$, and doubleheterozygous $\mathrm{Taz}^{+/-} ; \mathrm{Pkd1}^{+/-}$mice grown in osteoblastic differenti- 
A

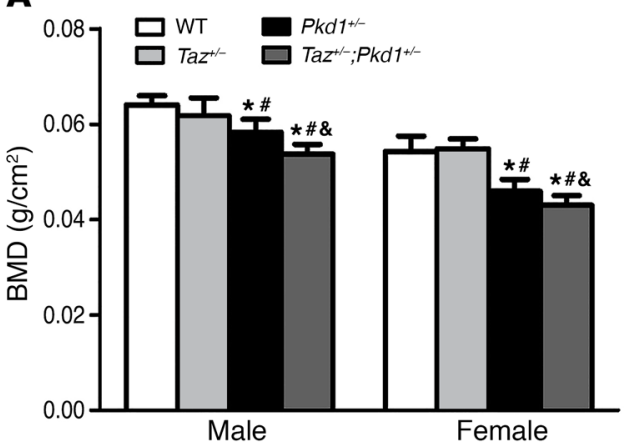

C

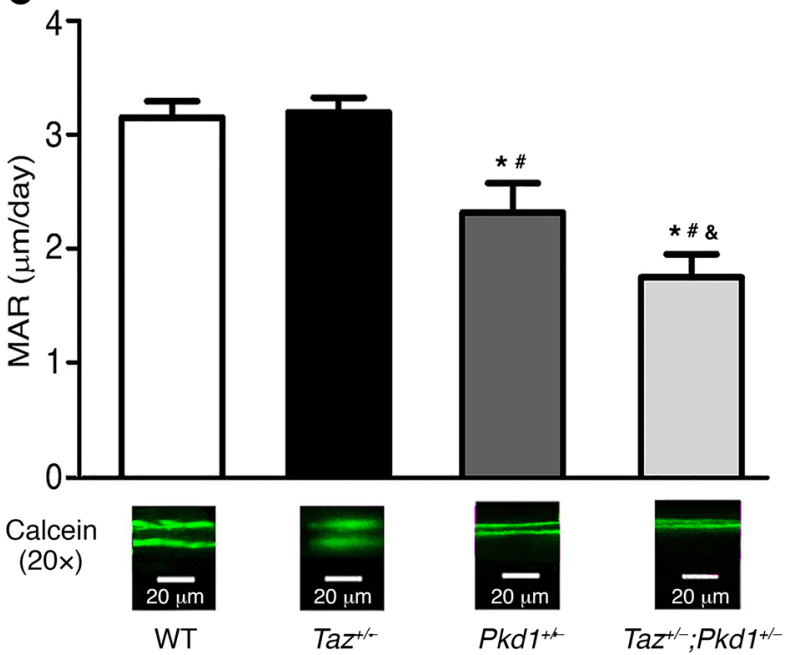

B

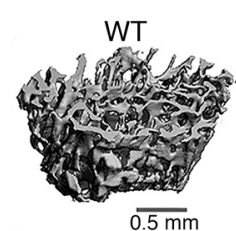

BV/TV

$\overline{0.5 \mathrm{~mm}}$
$32 \pm 2.8$

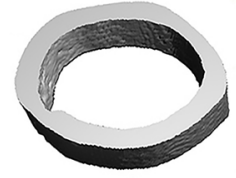

$0.5 \mathrm{~mm}$

Ct.Th

$0.187 \pm 0.011$

D

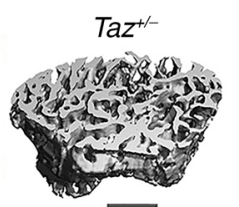

$0 . \overline{5 \mathrm{~mm}}$

$32 \pm 2.5$

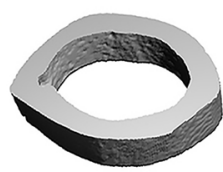

$\overline{0.5 \mathrm{~mm}}$

$0.189 \pm 0.116$

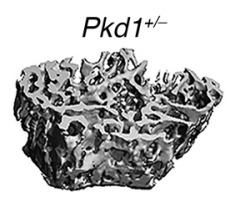

$\overline{0.5 \mathrm{~mm}}$

$27 \pm 2.2^{*}$

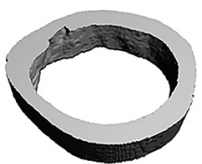

$\overline{0.5 \mathrm{~mm}}$

$0.169 \pm 0.115^{*}$

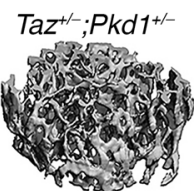

$0 . \overline{\mathrm{mm}}$

$23 \pm 3.5^{\text {* \# \& }}$

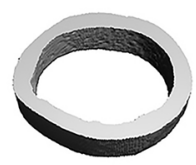

$\overline{0.5 \mathrm{~mm}}$

$0.150 \pm 0.010^{\star \# \&}$

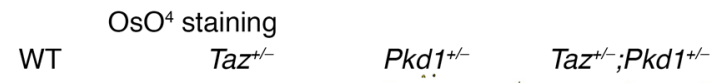

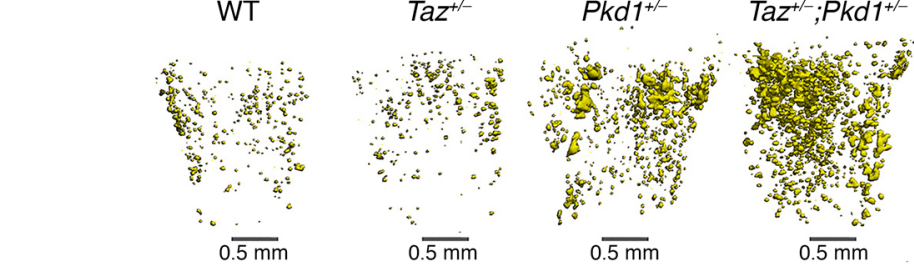

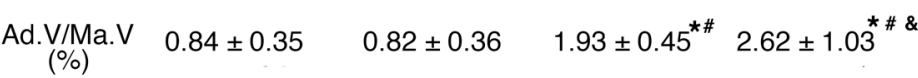

Ad.N

$16 \pm 6.8$

$16 \pm 7.0$

$3.7 \pm 8.6^{* \#}$

$50 \pm 18^{\text {*\# \& }}$

E

TRAP staining (10x)

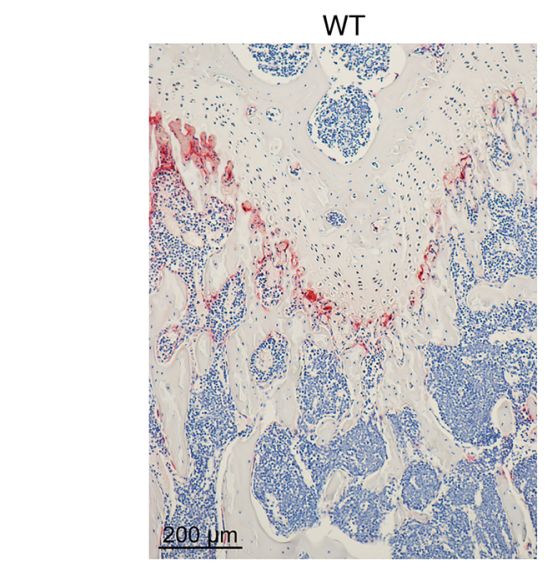

Oc.S/BS

$(\%)$

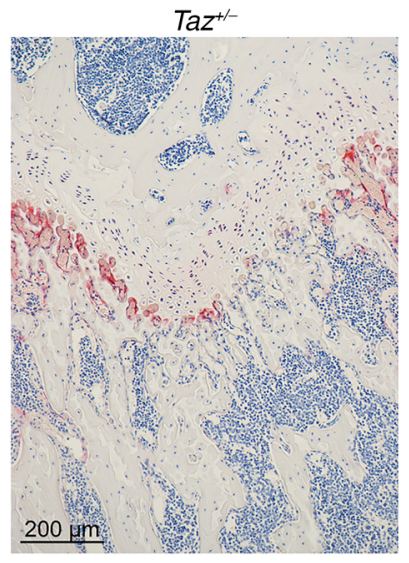

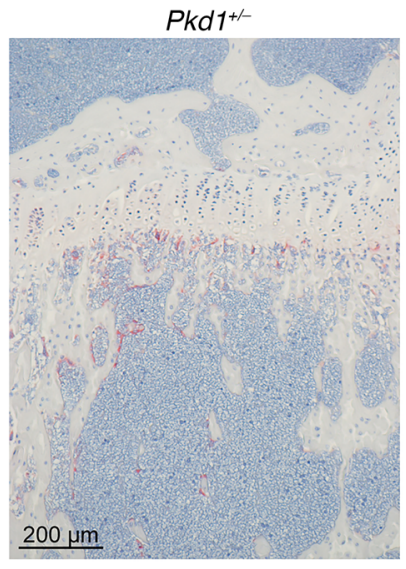

$5.2 \pm 0.55^{* \#}$

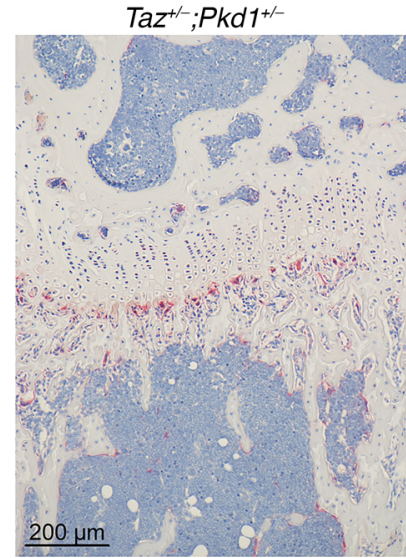

$5.4 \pm 0.38^{\star \#}$

Figure 1. Effects of combined Pkd1 and Taz deficiency on bone mass in 8-week-old mice. (A) BMD in femurs by dual-energy x-ray absorptiometry scan. (B) Micro-CT analysis of distal femoral metaphysis and midshaft diaphysis. (C) Periosteal mineral apposition rate (MAR) by calcein double labeling. (D) $\mathrm{OsO}_{4}$ staining of decalcified tibiae by micro-CT analysis. (E) TRAP staining (red color) for osteoclast activity. Data are presented as the mean \pm SD from 6-8 individual mice $(n=6-8)$. $P$ values were determined by 1-way ANOVA with Newman-Keuls multiple-comparisons test. ${ }^{*}$ Significant difference from WT control mice, " $s$ significant difference from Taz ${ }^{+-}$mice, \&significant difference from $P k d 1^{+/-}$mice at $P<0.05$, respectively. BV/TV, bone volume density; Ct.Th, cortical thickness; Ad.V/Ma.V, adipocyte volume per marrow volume; Ad.N, adipocyte number; Oc.S/BS, osteoclast surface per bone surface. 


\section{Table 1. Gene expression profiles in femurs from 8-week-old mice by real-time quantitative reverse transcription PCR}

\begin{tabular}{|c|c|c|c|c|c|}
\hline Gene & Accession no. & $\mathrm{Taz}^{+/-}$ & $\mathrm{Pkd1}^{+/-}$ & $\mathrm{Taz}^{+1-} ; \mathrm{Pkd1}^{+/-}$ & $P$ value \\
\hline \multicolumn{6}{|c|}{ Osteoblast lineage } \\
\hline Pkd1 & NM_013630.2 & $0.97 \pm 0.19$ & $0.50 \pm 0.17^{A, B}$ & $0.48 \pm 0.08^{A, B}$ & $<0.0001$ \\
\hline Taz & NM_133784.3 & $0.52 \pm 0.14^{A}$ & $1.02 \pm 0.32^{\mathrm{B}}$ & $0.50 \pm 0.13^{\mathrm{A}, \mathrm{C}}$ & $<0.0001$ \\
\hline Run $\times 2-11$ & NM_009820.5 & $1.05 \pm 0.22$ & $0.74 \pm 0.08^{A, B}$ & $0.60 \pm 0.16^{A, B}$ & 0.0007 \\
\hline Osteocalcin & NM_007541.2 & $0.99 \pm 0.26$ & $0.73 \pm 0.08^{A, B}$ & $0.47 \pm 0.06^{\mathrm{A}, \mathrm{B}, \mathrm{C}}$ & $<0.0001$ \\
\hline Osteopontin & AF515708.1 & $0.95 \pm 0.25$ & $0.72 \pm 0.12^{\mathrm{A}, \mathrm{B}}$ & $0.49 \pm 0.11^{A, B, C}$ & 0.0003 \\
\hline Mmp13 & NM_008607.2 & $0.93 \pm 0.15$ & $0.63 \pm 0.16^{A, B}$ & $0.41 \pm 0.11^{A, B, C}$ & $<0.0001$ \\
\hline Opg & NM_008764.3 & $0.94 \pm 0.20$ & $0.64 \pm 0.14^{A, B}$ & $0.67 \pm 0.16^{A, B}$ & 0.0048 \\
\hline RankL & NM_011613.3 & $0.94 \pm 0.27$ & $0.60 \pm 0.23^{A, B}$ & $0.67 \pm 0.20^{A, B}$ & 0.0033 \\
\hline Sost & NM_024449.6 & $0.99 \pm 0.16$ & $0.72 \pm 0.14^{A, B}$ & $0.55 \pm 0.09^{A, B, C}$ & $<0.0001$ \\
\hline Dmp1 & NM_016779.2 & $1.01 \pm 0.12$ & $0.73 \pm 0.16^{A, B}$ & $0.63 \pm 0.14^{A, B}$ & 0.0005 \\
\hline Wnt10b & U61970.1 & $1.10 \pm 0.18$ & $1.05 \pm 0.26$ & $0.95 \pm 0.15$ & 0.5621 \\
\hline Axin2 & AF205889.1 & $1.13 \pm 0.24$ & $1.19 \pm 0.42$ & $1.17 \pm 0.34$ & 0.6928 \\
\hline $\mathrm{Fzd} 2$ & NM_020510.2 & $1.15 \pm 0.29$ & $0.96 \pm 0.38$ & $0.99 \pm 0.17$ & 0.5539 \\
\hline \multicolumn{6}{|l|}{ Osteoclast } \\
\hline Trap & NM_007388.3 & $0.93 \pm 0.16$ & $0.65 \pm 0.06^{A, B}$ & $0.56 \pm 0.12^{\mathrm{A}, \mathrm{B}}$ & $<0.0001$ \\
\hline Mmpg & NM_013599.3 & $0.95 \pm 0.24$ & $0.71 \pm 0.05^{A, B}$ & $0.47 \pm 0.08^{A, B, C}$ & 0.0001 \\
\hline \multicolumn{6}{|l|}{ Chondrocyte } \\
\hline Type II collagen & NM_031163.3 & $1.02 \pm 0.45$ & $0.91 \pm 0.27$ & $0.94 \pm 0.13$ & 0.8916 \\
\hline VegfA & NM_009505.4 & $0.90 \pm 0.26$ & $1.03 \pm 0.24$ & $1.05 \pm 0.21$ & 0.8257 \\
\hline \multicolumn{6}{|l|}{ Adipocyte } \\
\hline Pparg & NM_009505.4 & $0.99 \pm 0.24$ & $1.51 \pm 0.39^{A, B}$ & $1.83 \pm 0.37^{A, B}$ & 0.0001 \\
\hline$a P 2$ & NM_024406.2 & $1.26 \pm 0.29$ & $1.71 \pm 0.28^{A}$ & $2.59 \pm 0.84^{A, B, C}$ & $<0.0001$ \\
\hline Lpl & NM_008509.2 & $1.17 \pm 0.20$ & $1.57 \pm 0.22^{A}$ & $2.19 \pm 0.77^{\mathrm{AB}, \mathrm{B}, \mathrm{C}}$ & 0.0001 \\
\hline
\end{tabular}

Data are mean \pm SD from 6 femurs of 8-week-old individual mice $(n=6)$ and are expressed as the fold changes relative to the housekeeping gene cyclophilin A subsequently normalized to control mice. $P$ values refer to the differences between group means by 1-way ANOVA. If the differences between the means are statistically significant (i.e., $P<0.05$ ), the group means are compared by Newman-Keuls multiple-comparisons test. ${ }^{A}$ Significant difference from WT control mice, ${ }^{\mathrm{B}}$ significant difference from $\mathrm{Taz}^{+/-}$mice, ${ }^{\mathrm{C}}$ significant difference from $\mathrm{Pkd1}^{+/-}$mice at $P<0.05$, respectively.

ation media for up to 12 days. Compared with age-matched WT and $\mathrm{Taz}^{+/-}$BMSCs, the $\mathrm{Pkd1}^{+/-}$cultures showed less abundant mineralized nodules and had significantly lower alizarin red S accumulation at day 12 of culture (Figure $2 \mathrm{~A})$. The $\mathrm{Pkd1}^{+/}$- cultures also displayed significantly lower alkaline phosphatase (ALPL) activity at day 8 of culture compared with age-matched WT (Figure 2B). In addition, the $\mathrm{Taz}^{+/} ; \mathrm{Pkd1}^{+/-}$cultures had greater reductions in mineralization accumulation and ALPL activity.

To investigate whether combined $T A Z$ and $P k d 1$ deficiency resulted in additive effects on gene expression profiles in BMSC osteogenic cultures, we examined by real-time RT-PCR the expression levels of a panel of osteoblast- and adipocyte-related mRNAs at day 8 of culture. Significantly greater reductions of osteoblastrelated genes were observed in double-heterozygous $\mathrm{Taz}^{+/-} ; \mathrm{Pkd1}^{+/-}$ BMSC cultures for Runx2 and osteocalcin, whereas much greater increments of adipocyte-related genes were observed in doubleheterozygous $\mathrm{Taz}^{+-} ; \mathrm{Pkd1}^{+/-}$mice for Pparg and $\mathrm{AP} 2$ compared with age-matched heterozygous $P k d 1^{+/-}$cultures (Figure 2C).

Similar findings were observed in $P k d 1$-deficient osteoblasts grown under conditions that promote osteoblast differentiation. We observed that loss of $P k d 1$ in primary osteoblasts resulted in significant reductions in osteogenic markers such as Runx2 and osteocalcin and enhanced adipogenic markers such as Pparg and $a P 2$ in vitro (Supplemental Figure 1A; supplemental material available online with this article; https://doi. org/10.1172/JCI93725DS1). In contrast, Pkd2deficient osteoblasts exhibited coordinated reductions in both osteogenic and adipogenic markers during culture under osteogenic conditions (Supplemental Figure 1B). To verify these results, and to remove any confounding effects of the in vivo environment on the phenotype of osteoblasts isolated from knockout mice, we used adenovirus-CMV-iCre (Ad-CMV-iCre) and freshly isolated primary osteoblasts derived from the floxed $P k d 1^{f / f l}$ and $P k d 2^{f / f l}$ mice to examine whether loss of polycystins modulates osteoblast and adipocyte differentiation. We found that incubation of primary $P k d 1^{f / f l}$ and $P k d 2^{f / f l}$ osteoblasts with Ad-CMV-iCre ex vivo resulted in a 65\% decrease in $P k d 1$ and $P k d 2$ message expression compared with Ad-CMV-null vector controls. The reduction in $P k d 1$ and $P k d 2$ transcripts resulted in significant reductions of osteogenic markers, such as Runx2 and alkaline phosphatase $(A l p l)$ (Supplemental Figure 1C). However, Ad-CMV-iCre-mediated deletion of $P k d 1$ resulted in significant increases of adipogenic markers, such as Pparg and aP2 (Supplemental Figure 1C), whereas Ad-CMV-iCre-mediated deletion of $P k d 2$ ex vivo resulted in decreased adipogenic markers (Supplemental Figure 1D), consistent with observations in conditional $P k d 1^{O c-c K O}$ and $P k d 2^{O c-c K O}$ mice as we previously reported (30-32).

Evidence for a functional link between polycystins and TAZ in vitro. We assessed changes in TAZ protein expression and phosphorylation in Pkd1- and Pkd2-deficient osteoblasts. We found that the level of total TAZ protein was slightly decreased, but TAZ phosphorylation at Ser 89, which leads to TAZ cytoplasmic sequestration (47), was increased in both Pkd1- and Pkd2-deficient osteoblasts (Figure 3, A and B). Consistent with reductions in functional TAZ protein levels, we observed that TAZ-mediated activation of TEAD reporter, a measure of TAZ transcriptional activity, was markedly decreased in both $P k d 1$ - and $P k d 2$-deficient osteoblasts (Figure 3C). Although both $P k d 1$ and $P k d 2$ deficiencies lead to decreased TEAD reporter activities, they may differentially regulate TAZ.

To explore TAZ interactions with PC1 and PC2, we overexpressed FLAG-tagged TAZ and full-length PC1 and PC2 in HEK-293T cells and performed coimmunoprecipitation (co-IP) with an anti-FLAG antibody. We found that TAZ forms a complex with PC1 and PC2, as evidenced by their co-IP (Figure 3D). We also substituted PC1-CTT for the full-length PC1 in these studies. We found that PC1-CTT was sufficient for binding to TAZ, since overexpression of PC1-CTT brought down TAZ and PC2 in the IP complex with an anti-myc antibody for PC1-CTT (Figure 3D). To test whether polycystins regulate TAZ function, we cotrans- 
A

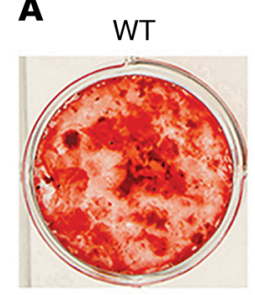

$136 \pm 6.0$

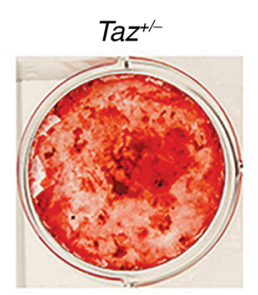

Alizarin red-S ( $\mu \mathrm{mol} / \mathrm{ug}$ protein)

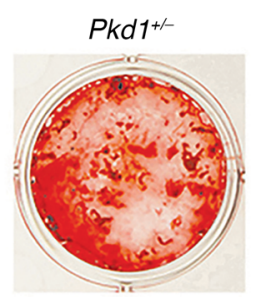

$97 \pm 5.3^{* \#}$

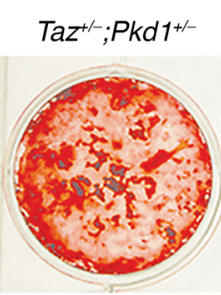

$77 \pm 13.5^{* \# \&}$
B

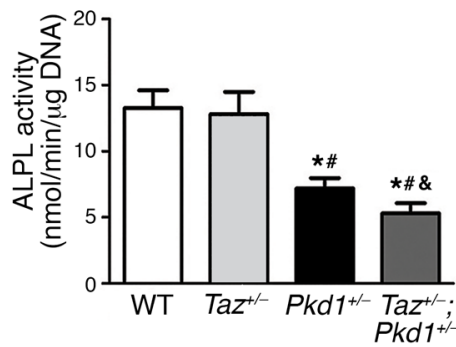

c

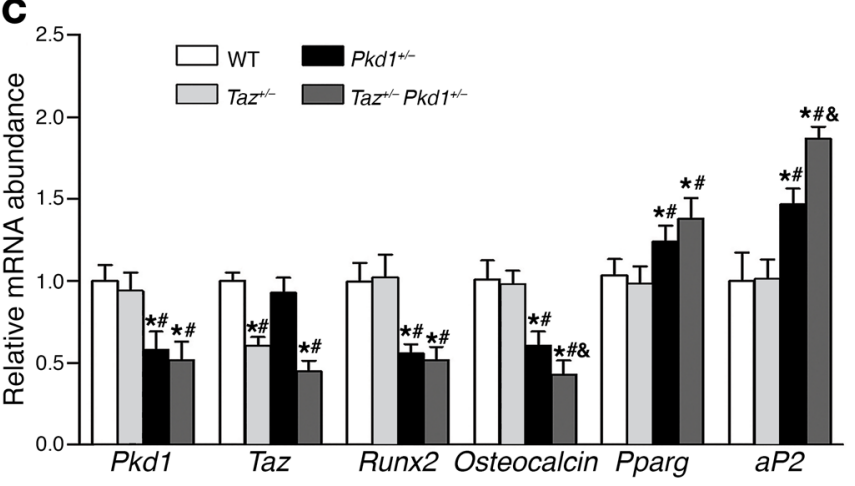

fected either full-length $P C 1$ or PC1-CTT and full-length PC2 along with TEAD reporter constructs into C3H1OT1/2 cells. We found that overexpression of either full-length PC1 or PC1-CTT along with full-length $P C 2$ markedly increased TAZ-induced activation of TEAD activity (Figure 3E), suggesting a functional link between polycystins and TAZ signaling. These findings are also consistent with the observations that PC2 increases PC1 C-terminal cleavage in COS-7 cells $(48,49)$, and subsequently enhances PC1-CTT nuclear translocation and TAZ signaling pathway.

$P C 1$ C-tail interacts with TAZ to coregulate Runx 2 and PPAR $\gamma$ activities. Next, we examined whether the PC1-CTT/TAZ complex coregulates Runx2 and PPAR $\gamma$ activities $(33,42)$. It is known that the PC1-CTT binds to TAZ, which is a coactivator for Runx2 and a corepressor for PPAR $\gamma$ activity $(42,44)$, but the effects of the PC1CTT/TAZ complex on Runx2 and PPAR $\gamma$ activities have not been studied. Thus, we overexpressed FLAG-tagged TAZ, PC1-CTT, and Runx2 or Pparg in HEK-293T cells and performed co-IP with an anti-FLAG antibody (Figure 4, A and B). We found that TAZ was sufficient for binding to PC1-CTT, since overexpression of PC1-CTT brought down TAZ in the IP complex. We also found that TAZ and PC1-CTT form a complex with either Runx2 or PPAR $\gamma$, as evidenced by their co-IP (Figure 4, A and B). Overexpression of TAZ, PC1-CTT, and Runx2 or PPAR $\gamma$ in multipotent C3H10T1/ 2 mesenchymal cells found that TAZ stimulated osteocalcin $(O c)$ promoter activity and was enhanced by PC1-CTT, and overexpression of Runx2. In contrast, we observed that TAZ inhibits PPAR $\gamma$ stimulation of $a P 2$ promoter activity, and this effect was further suppressed by overexpression of PC1-CTT (Figure 4, C and D). These findings suggest that PC1-CTT modulates TAZ function through its binding to TAZ, which coactivates Runx2-mediated gene transcription and corepresses PPAR $\gamma$-induced gene expression.

To examine the effects of PC1 C-tail cleavage on $O c$ and $a P 2$ promoter reporter activities, we overexpressed membrane-bound
Figure 2. Assessment of osteoblastic differentiation in bone marrowderived mesenchymal stem cell cultures from 8-week-old WT, $\mathrm{Taz}^{+/-}$, and $\mathbf{P k d 1}^{+/-}$mutant mice. (A) Histochemical staining of mineralization nodules with alizarin red $S$ during 12 -day cultures. (B) ALPL activity during 8-day cultures. (C) Osteogenic and adipogenic gene expressions during 8-day cultures. Data are presented as the mean \pm SD from 3 independent experiments $(n=3)$. $P$ values were determined by 1-way ANOVA with Newman-Keuls multiple-comparisons test. ${ }^{*}$ Significant difference from WT control mice, "significant difference from $\mathrm{Taz}^{+/-}$mice, ${ }^{\star}$ significant difference from $P k d 1^{+/}$mice at $P<0.05$, respectively.

$P C 1$-CTT construct $(26,50)$ along with $O c$ or $a P 2$ reporters in C3H10T1/2 cells. Overexpression of PC1-CTT stimulated Oc promoter reporter activities, but suppressed $a P 2$ promoter reporter activities (Figure 4, E and F). As previously reported (51), PC1-CTT is released by a $\gamma$-secretase. We found that DAPT, a $\gamma$-secretase inhibitor, blocked the effects of PC1-CTT to stimulate $O c$ promoter, and reversed the inhibitory effect on $a P 2$ promoter activities (Figure 4, E and F). These findings suggest that cleavage and release of the PC1 C-tail from the membrane form that translocates to the nucleus play an essential role in the regulation of targeting gene transcription.

Next, we investigated the co-occupancy of endogenous $O c$ or aP2 promoter by PC1-CTT, TAZ, and Runx2 or PPAR $\gamma$ complex in C3H1OT1/2 cells. OSE2 is a key osteoblast-specific cis-acting element in the proximal Oc promoter (52); Runx 2 was shown to bind the OSE2 site and regulates mouse Ocpromoter activity (53). Therefore, we examined whether PC1-CTT, TAZ, and Runx2 form a complex that binds the region of the proximal $O c$ promoter that contains the OSE2 site. Quantitative chromatin immunoprecipitation (ChIP) analyses using anti-FLAG for TAZ, anti-myc for PC1-CTT, and antiRunx2 identified that TAZ, PC1-CTT, and Runx2 were specifically recruited to a region of the proximal $O c$ promoter containing the known OSE2 cis-element that is known to bind to Runx2 (Figure $4 \mathrm{G}$ ). There was an approximately 3 - to 7 -fold increase in the ratio of the promoter sequence versus the coding region sequence in the anti-FLAG, anti-myc, or anti-Runx2 group compared with the IgG control group by quantitative real-time PCR. To establish that TAZ and PC1-CTT suppress PPAR $\gamma$ binding to the consensus ARE6 site in the proximal aP2 promoter (54), we performed quantitative ChIP analysis using an anti-FLAG for TAZ, an anti-myc for PC1-CTT, and an anti-PPAR $\gamma$ antibody. Real-time PCR on the immunoprecipitated DNA fragments with primers to amplify the region spanning the ARE6 sites con- 
A

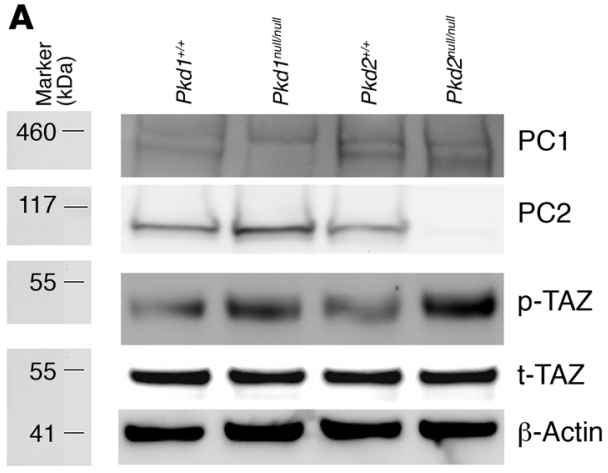

B

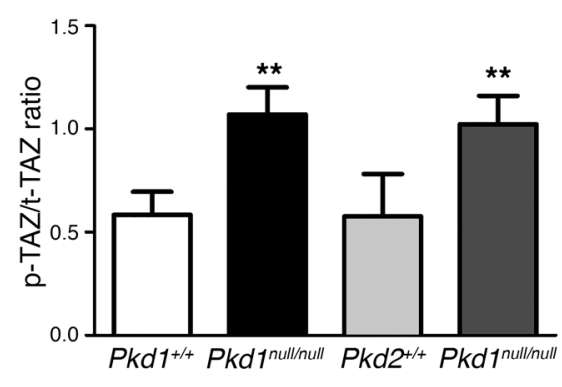

C

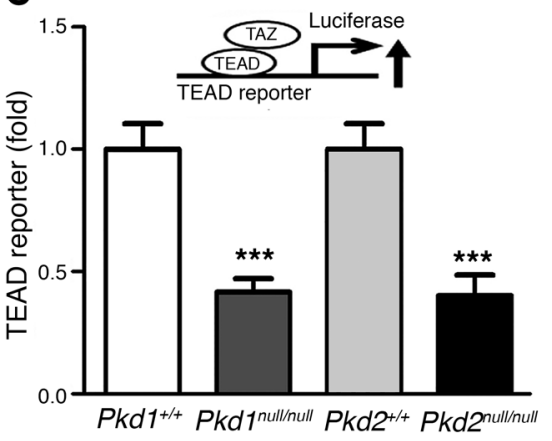

E

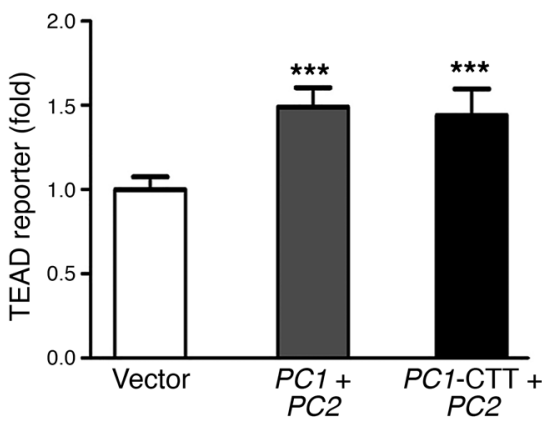

D
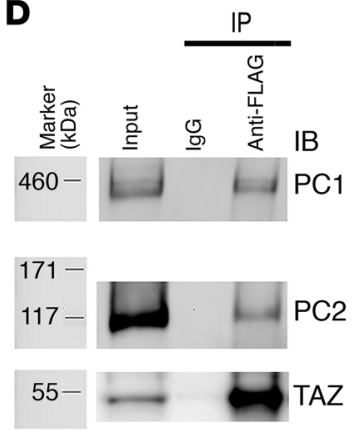
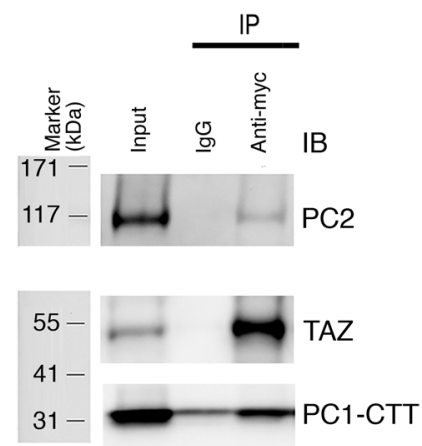

Figure 3. A functional link between polycystins and TAZ signaling. (A) Western blot analysis of TAZ protein and its phosphorylation in both $P k d 1$ - and $P k d 2$-deficient osteoblasts. (B) The quantification of phosphorylated TAZ ( $p$-TAZ) abundance relative to that of total TAZ ( $t-T A Z$ ) in cultured osteoblasts. (C) TAZ-mediated activation of TEAD reporter activities in Pkd1- or Pkd2-deficient osteoblasts. (D) Co-IP analysis of full-length PC1, full-length PC2, PC1-CTT, and TAZ protein interaction in HEK-293T cells. (E) Effects of full-length PC1, full-length PC2, and PC1-CTT on TAZ-mediated activation of TEAD reporter activities in $\mathrm{C} 3 \mathrm{H} 10 \mathrm{~T} 1 / 2$ cells. Data are presented as the mean $\pm \mathrm{SD}$ from 3 independent experiments $(n=3)$. $P$ values were determined by 1-way ANOVA with Newman-Keuls multiple-comparisons test. ${ }^{* *} P<0.01,{ }^{* *} P<0.001$ compared with control group.

firmed that TAZ, PC1-CTT, and PPAR $\gamma$ were specifically recruited to the segment of the proximal aP2 promoter containing the ARE6 site that is known to bind PPAR $\gamma$ (Figure $4 \mathrm{H})$. There was an approximately 3- to 7-fold increase in the ratio of the promoter sequence versus the coding region sequence in the anti-FLAG, anti-myc, or anti-PPAR $\gamma$ group compared with the IgG control group by quantitative real-time PCR. Together, these findings indicate that PC1CTT and TAZ colocalize to the proximal Oc promoter to enhance Runx2-mediated osteocalcin gene transcription, and colocalize to the proximal aP2 promoter to enhance PPAR $\gamma$-mediated $a P 2$ gene transcription.

Matrix stiffness regulates Oc or aP2 promoter activities by PC1CTT and TAZ nuclear translocation. Previous publications showed that matrix stiffness has an important impact on osteogenesis and adipogenesis (33). We examined whether matrix stiffness affects PC1-CTT and TAZ nuclear translocation to regulate osteogenic $O c$ promoter reporter and adipogenic aP2 promoter reporter activities in $\mathrm{C} 3 \mathrm{H} 10 \mathrm{~T} 1 / 2$ cells transfected with PC1CTT. Using C-terminal FLAG-tagged full-length PC1 construct, we found that hard matrix promotes $\gamma$-secretase and PC1-CTT cleavage, whereas soft matrix inhibits $\gamma$-secretase and PC1-CTT cleavage (Figure 5, A and B). Using membrane-bound PC1-CTT construct, we observed that hard matrix $(40 \mathrm{kPa})$ promotes PC1CTT and TAZ translocation to the nucleus (Figure 5, C and D) and increases $O c$ promoter reporter activity (Figure $5 \mathrm{E}$ ), whereas soft matrix $(0.5 \mathrm{kPa})$ attenuates PC1-CTT and TAZ translocation to nucleus (Figure 5, C and D) and enhances aP2 promoter reporter activity (Figure 5F). In addition, DAPT, a $\gamma$-secretase inhibitor, blocked the effects of the transfected PC1-CTT to stimulate $O c$ promoter reporter, and reversed the inhibitory effect on $a P 2$ promoter reporter activities (Figure 5, E and F). We observed that the TEAD, Oc, and $a P 2$ promoter activity responded similarly in C3H1OT1/2 cells expressing endogenous PC1 as compared with cells transfected with PC1-CTT (Supplemental Figure 2, A-C). In addition, real-time RT-PCR showed that soft matrix inhibited the expression of the osteogenic genes Runx2, $A l p l$, and osteocalcin but promoted the expression of the adipogenic markers Pparg and aP2 in C3H1OT1/2 cells, whereas hard matrix had the opposite effect on gene expression, which was reversed by treatment with the $\gamma$-secretase inhibitor 
A

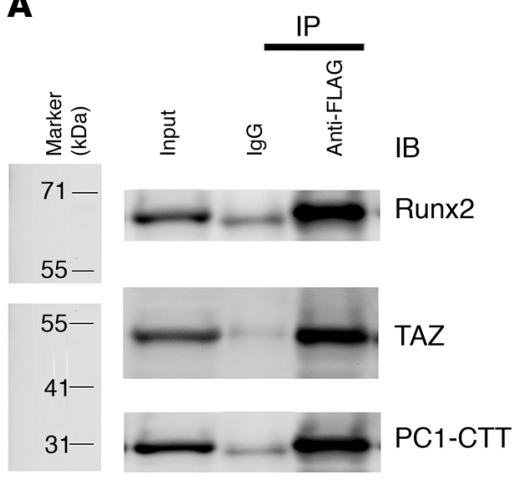

C

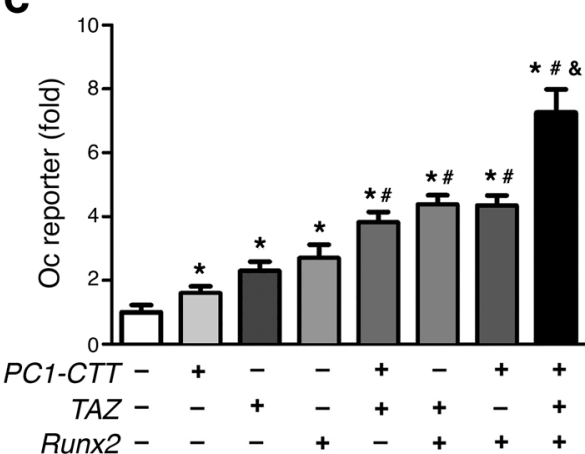

E

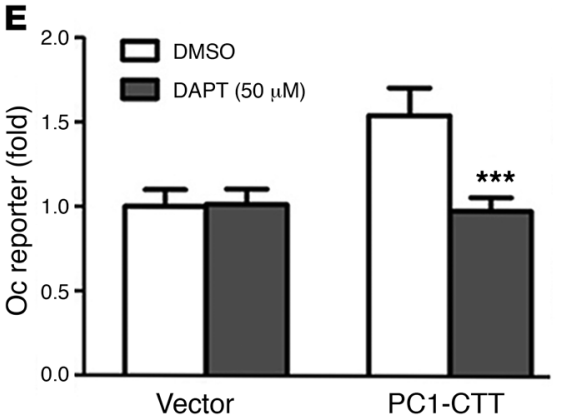

G

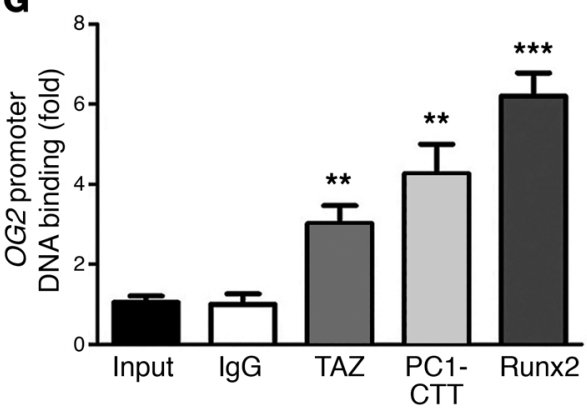

B

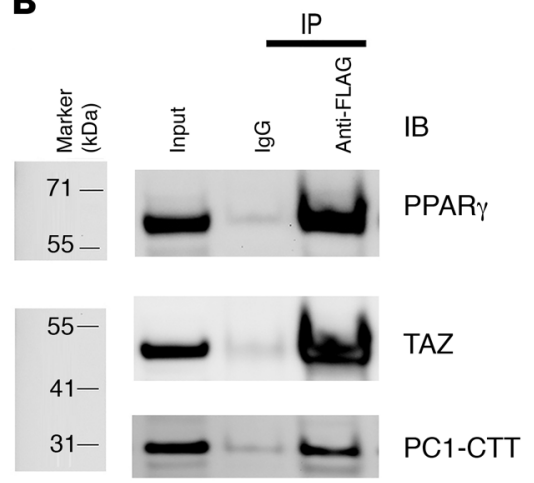

D

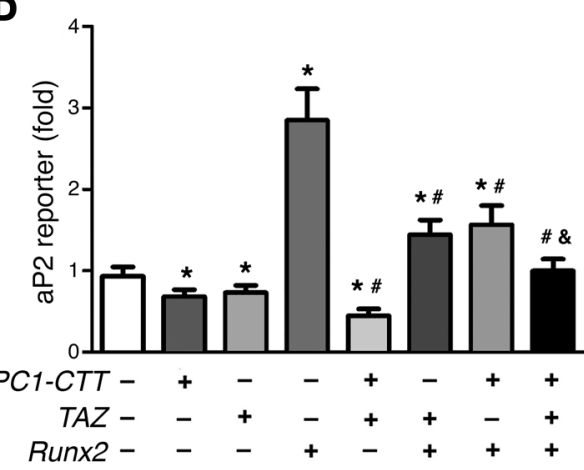

F

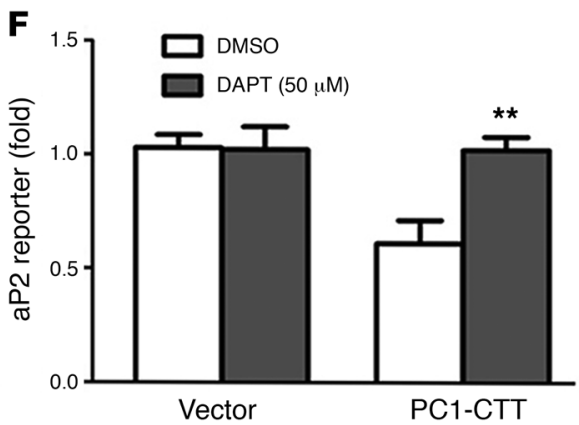

H

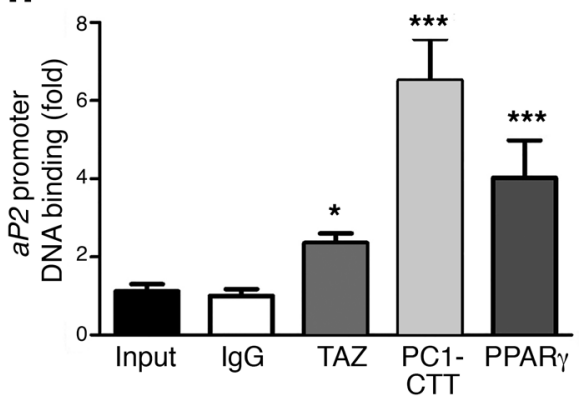

Figure 4. TAZ and PC1-CTT interaction differentially regulates Runx2 and PPAR $\gamma$ activities in C3H10T1/ 2 cells. (A and B) TAZ and PC1-CTT form complexes with Runx2 or PPAR $\gamma$. (C and D) TAZ promotes PC1-CTT-stimulated Runx2-mediated osteocalcin $(O c)$ promoter reporter activity but inhibits PPAR $\gamma$-induced $a P 2$ promoter reporter activity. (E and $\mathbf{F}$ ) Effects of the $\gamma$-secretase inhibitor DAPT on PC1-CTT-mediated Oc reporter and $a P 2$ reporter activities. ( $\mathbf{G}$ and $\mathbf{H}$ ) Quantitative ChIP analysis of co-occupancy of transcription factors in the endogenous Oc promoter-containing OSE2 site or the aP2 promoter-containing ARE6 site. Data are presented as the mean \pm SD from 3 independent experiments $(n=3)$. $P$ values were determined by 1-way ANOVA with Newman-Keuls multiple-comparisons test. ${ }^{*} P<0.05,{ }^{* *} P<0.01,{ }^{* *} P<0.001$ compared with control group. "Significant difference from either PC1-CTT, TAZ, or Runx2 or Pparg alone; \&significant difference from $P C 1-C T T$ plus TAZ, PC1CTT plus Runx2, TAZ plus Runx2, PC1-CTT plus Pparg, or TAZ plus Pparg cDNA constructs at $P<0.05$, respectively.
(DAPT) (Supplemental Figure 2D). These findings suggest that matrix stiffness regulates cleavage, release, and nuclear translocation of PC1-CTT from plasma membrane in the regulation of targeting gene transcription.

Mechanical stretch regulates Oc or aP2 promoter activities by PC1-CTT and TAZ nuclear translocation. Previous studies show that mechanical stretch promotes osteogenesis (55-57) and inhibits adipogenesis $(1,5,58)$. We examined whether stretch affects PC1-CTT cleavage and TAZ nuclear translocation to reg- ulate osteogenic $O c$ promoter reporter and adipogenic $a P 2$ promoter reporter activities in $\mathrm{C} 3 \mathrm{H} 10 \mathrm{~T} 1 / 2$ cells. Using C-terminal FLAG-tagged full-length PC1, we found that stretch stimulates $\gamma$-secretase activity and PC1-CTT cleavage, whereas the $\gamma$-secretase inhibitor DAPT abolished stretch-induced PC1-CTT cleavage (Figure 6, A and B). Using membrane-bound PC1-CTT, we observed that stretch promotes PC1-CTT and TAZ translocation to the nucleus (Figure 6, C and D) and increases TEAD and Oc promoter reporter activities (Figure 6, E and F), whereas DAPT blocks PC1- 
A

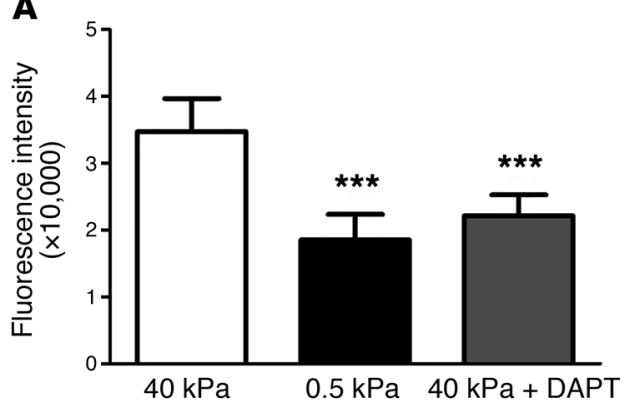

B

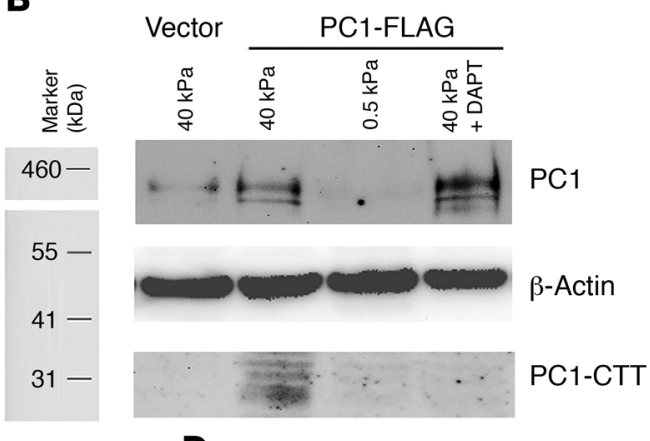

D
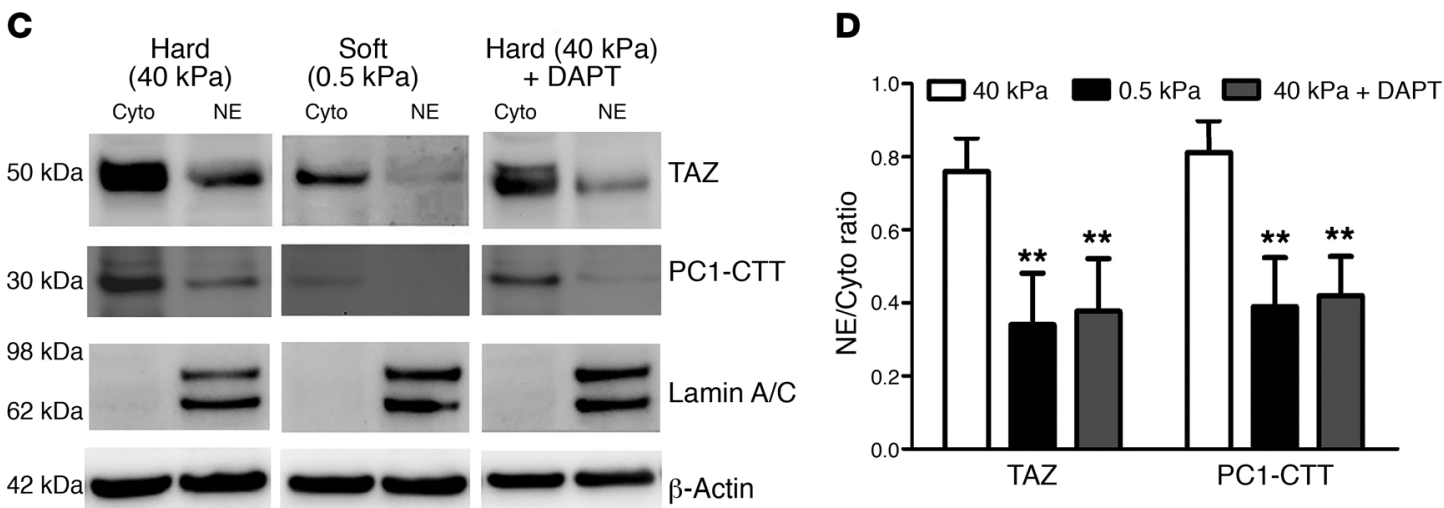

E

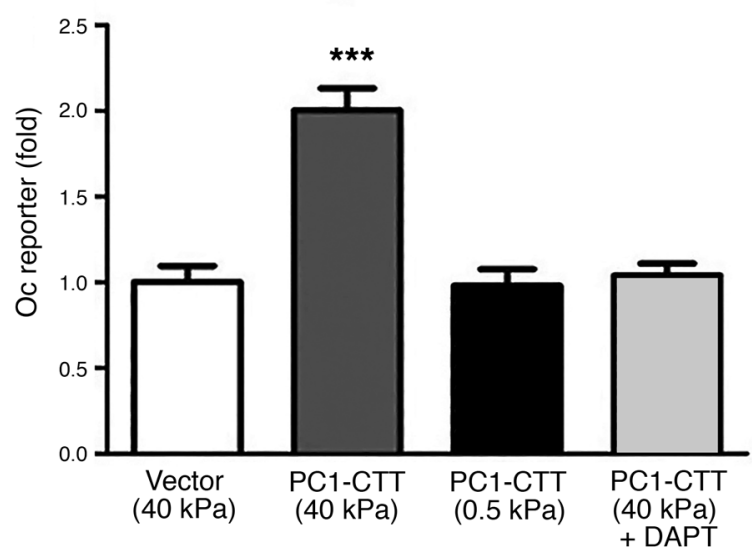

F

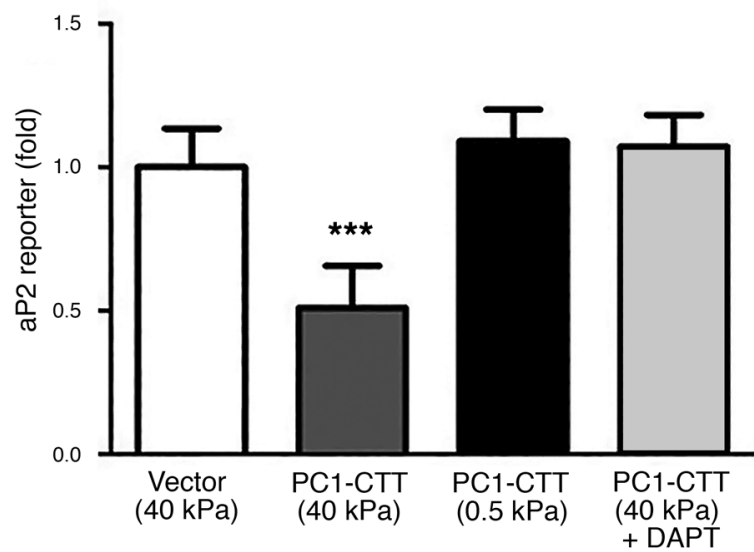

Figure 5. Matrix stiffness regulates Oc or aP2 promoter activities through PC1-CTT cleavage and TAZ nuclear translocation in C3H10T1/2 cells. (A) $\gamma$-Secretase activity. (B) A cleavage of PC1-CTT from full-length PC1. (C) Western blot analysis of cytoplasmic (Cyto) and nuclear-extracted (NE) proteins. (D) The quantification of NE TAZ or PC1-CTT abundance relative to that of Cyto TAZ or PC1-CTT in C3H10T1/2 cells transfected with PC1-CTT constructs. (E) $O c$ promoter reporter activity. (F) $a P 2$ promoter reporter activity. Data are presented as the mean \pm SD from 3 independent experiments $(n=3)$. $P$ values were determined by 1-way ANOVA with Newman-Keuls multiple-comparisons test. ${ }^{* *} P<0.01,{ }^{* *} P<0.001$ compared with control group.

CTT and TAZ translocation to nucleus (Figure 6, C and D) and enhances $a P 2$ promoter reporter activity (Figure 6G). Real-time RT-PCR confirmed that stretch enhanced the expression of the osteogenic genes Runx2, Alpl, and osteocalcin but inhibited expression of the adipogenic markers Pparg and aP2 (Supplemental Figure 3). These findings suggest that mechanical stretch stimulates osteogenesis but inhibits adipogenesis through regulation of PC1CTT and TAZ nuclear translocation.

Discovery of a small molecule that activates PC1/PC2/TAZ signaling. We constructed a 3D model of the PC1:PC2 coiled-coil structure based on previous published structural information (17) and performed docking calculations $(59,60)$ to identify compounds predicted to bind to the $\mathrm{PC} 2 \mathrm{PC} 1$ interacting region. From this screen of compounds predicted to modulate protein:protein interactions, Zinc01442821 (4-phenyl-1H-pyrrole-3-carboxylic acid, designated MS for molecular staple) was the best compound predicted to bind to the $\alpha$ regions of PC2, as shown in Figure 7A. In the computational model, Zinc01442821 binding forms hydrophobic interactions with $\mathrm{Val}^{1800}$ and Leu ${ }^{881}$ that are essential for the PC1-PC2 coiled-coil stabilization and also with charged PC2 residues $\mathrm{Arg}^{877}, \mathrm{Arg}^{878}$, and Lys ${ }^{874}$, i.e., with the central region of the KRRE cluster that is essential to the PC1:PC2 interactions (Figure 7B) (17).

To assess engagement of Zinc01442821 with polycystins and TAZ, we performed co-IP assays using HEK-293T cell lysates 
A

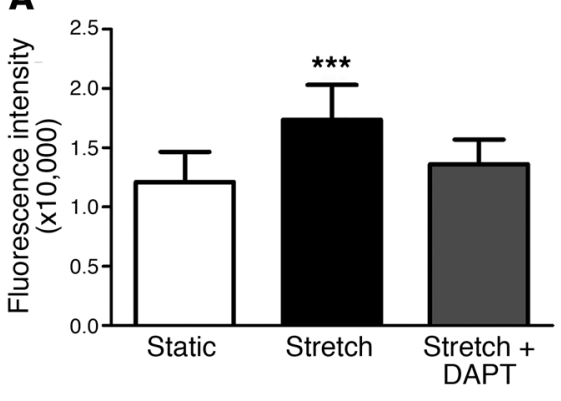

B

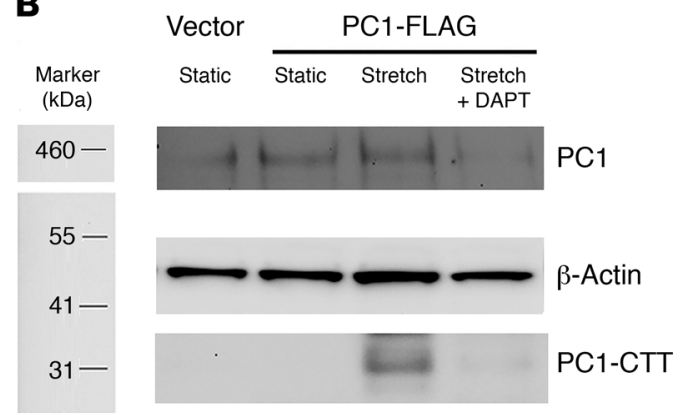

D

C

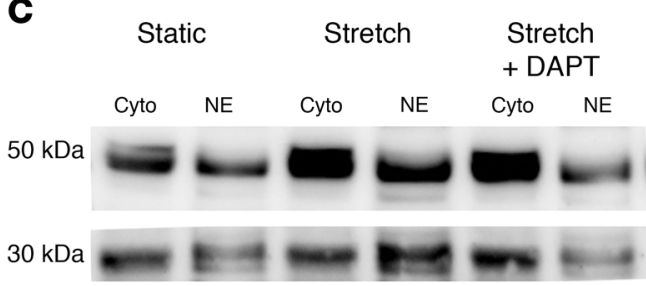
TAZ P1-CTT

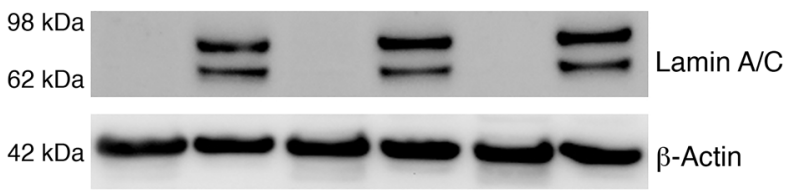

E

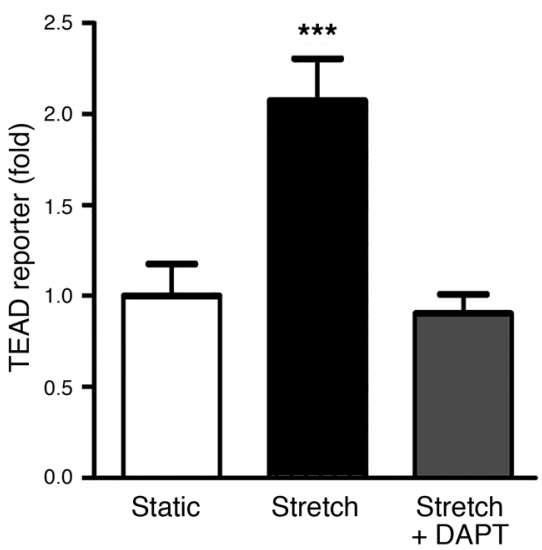

$\mathbf{F}$

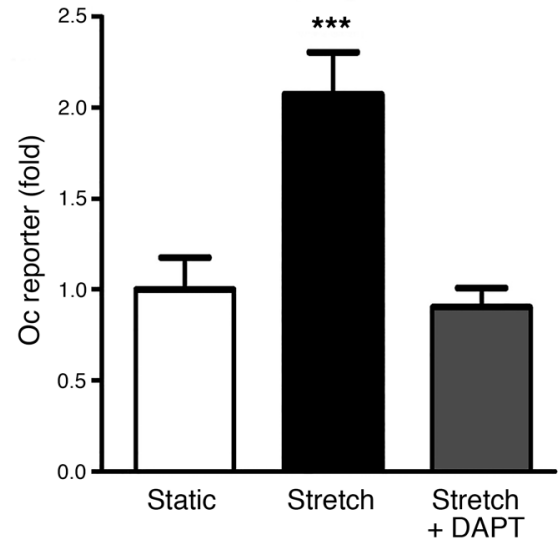

G

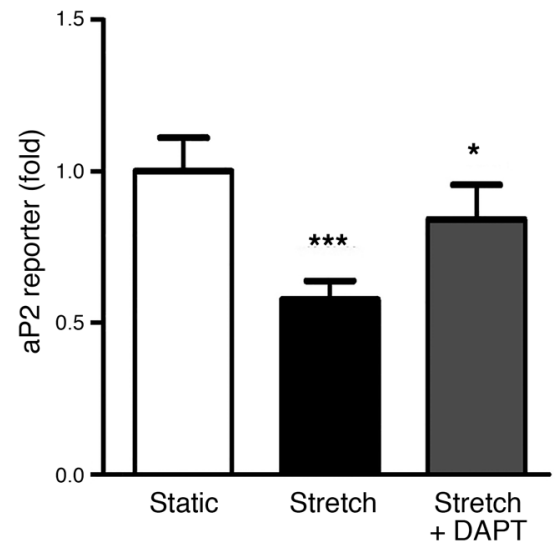

Figure 6. Effects of mechanical stretch on $\gamma$-secretase activity, PC1-CTT cleavage, TAZ nuclear translocation, TAZ-mediated TEAD reporter, and Oc and $\boldsymbol{a P 2}$ promoter reporter activities in C3H10T1/2 cells. (A) $\gamma$-Secretase activity. (B) A cleavage of PC1-CTT from FLAG-tagged full-length PC1. (C) Western blot analysis of cytoplasmic (Cyto) and nuclear-extracted (NE) proteins. (D) The quantification of NE TAZ or PC1-CTT abundance relative to that of Cyto TAZ or PC1-CTT in C3H10T1/2 cells transfected with PC1-CTT. (E) TEAD reporter activity. (F) Oc promoter reporter activity. (G) aP2 promoter reporter activity. Data are presented as the mean \pm SD from 3 independent experiments $(n=3)$. P values were determined by 1 -way ANOVA with Newman-Keuls multiplecomparisons test. ${ }^{*} P<0.05,{ }^{* *} P<0.001$ compared with control group.

cotransfected with GFP-tagged full-length PC1, full-length PC2, and FLAG-tagged TAZ. Cells were treated with either Zinc01442821, its inactive analog MS4 (see below), or the DMSO vehicle control. We found that the FLAG antibody coprecipitated PC1, PC2, and TAZ in vehicle-treated cultures, consistent with the above studies showing that PC1, PC2, and TAZ form a trimeric complex (Figure 3). The addition of Zinc01442821 (10 $\mu \mathrm{M})$ attenuated the incorporation of TAZ into this trimeric complex (Figure 7, C-E), but enhanced the co-IP of PC1 and PC2 complexes (Figure 7, F-H), consistent with stabilization of PC1 and PC2 interactions.

Using an MC3T3-E1 osteoblastic cell line that expresses endogenous PC1/PC2, we tested the effects of Zinc01442821 on intracellular calcium and TAZ-mediated activation of the TEAD reporter. We observed that Zinc01442821 significantly increased the peak value of Fluo-4 intensity and intracellular calcium levels (Figure 8A), consistent with activation of PC1/PC2 signaling. In addition, Zinc01442821 significantly increased TEAD reporter activity, consistent with enhanced TAZ nuclear translocation (Figure 8B) in MC3T3-E1 osteoblastic cells. The above co-IP data and these signaling responses suggest that Zinc01442821 may interact with PC2 to enhance calcium signaling as well as disrupt PC1/TAZ interactions to promote TAZ signaling.

Next, we examined the dose-dependent effects of Zinc01442821 on osteoblastogenesis and adipogenesis in 
A

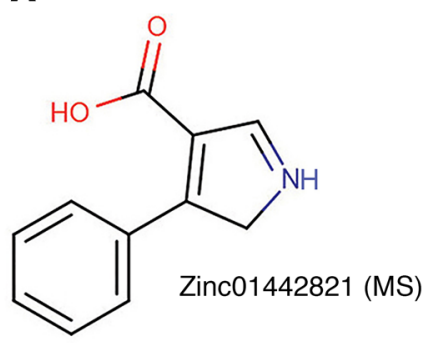

B

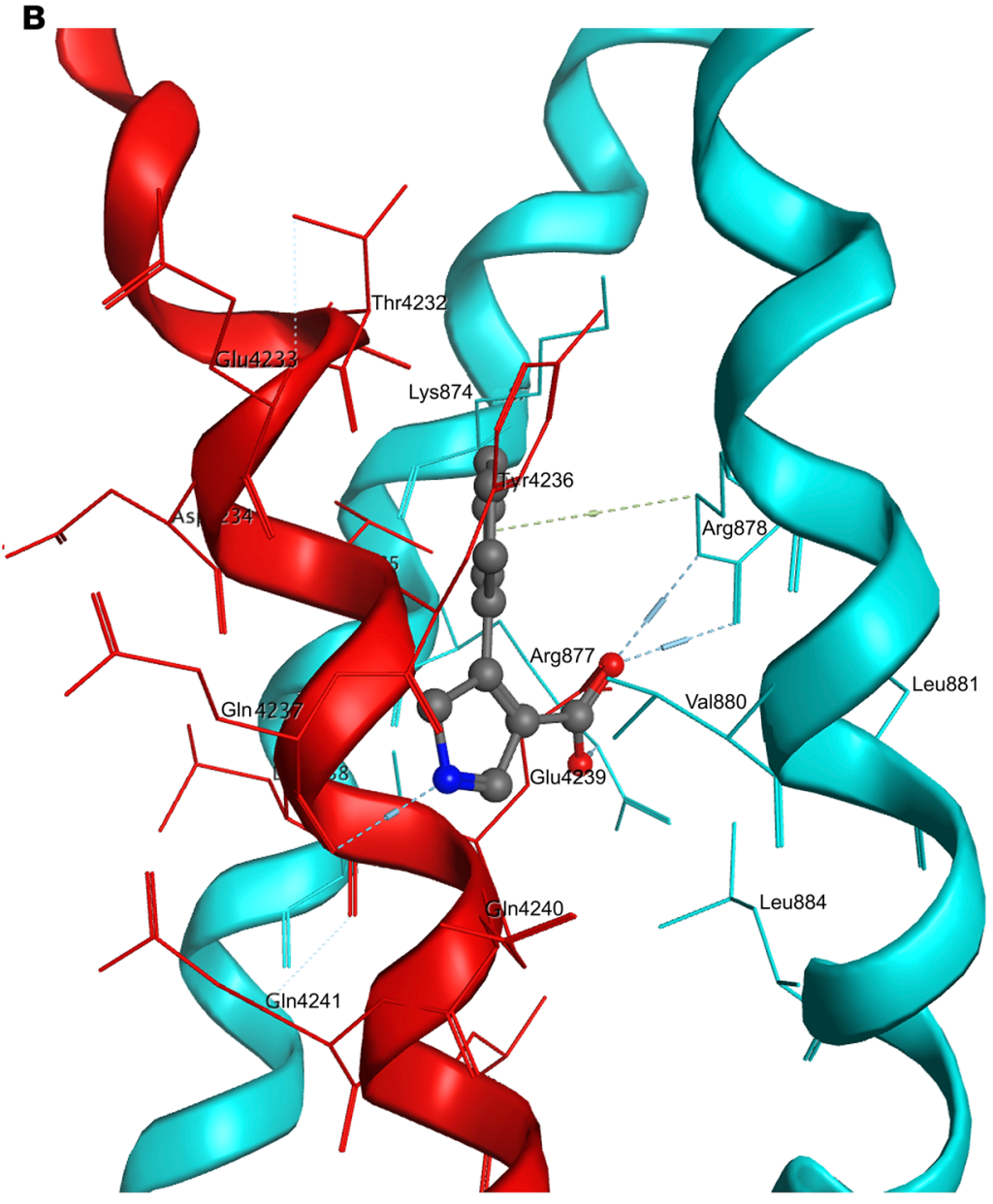

D

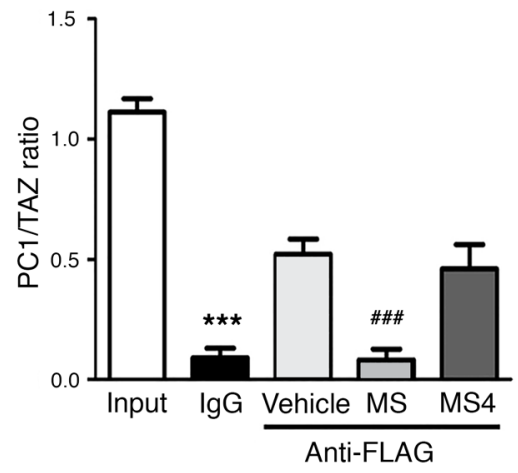

E

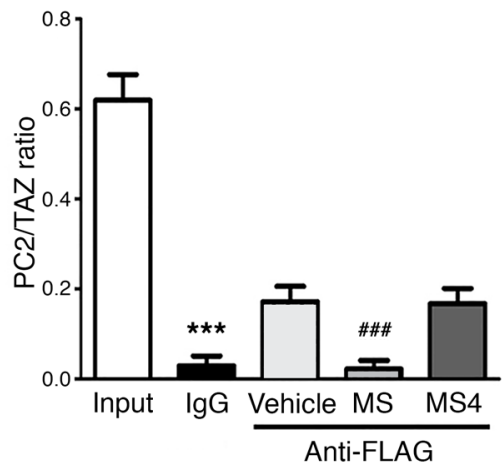

H
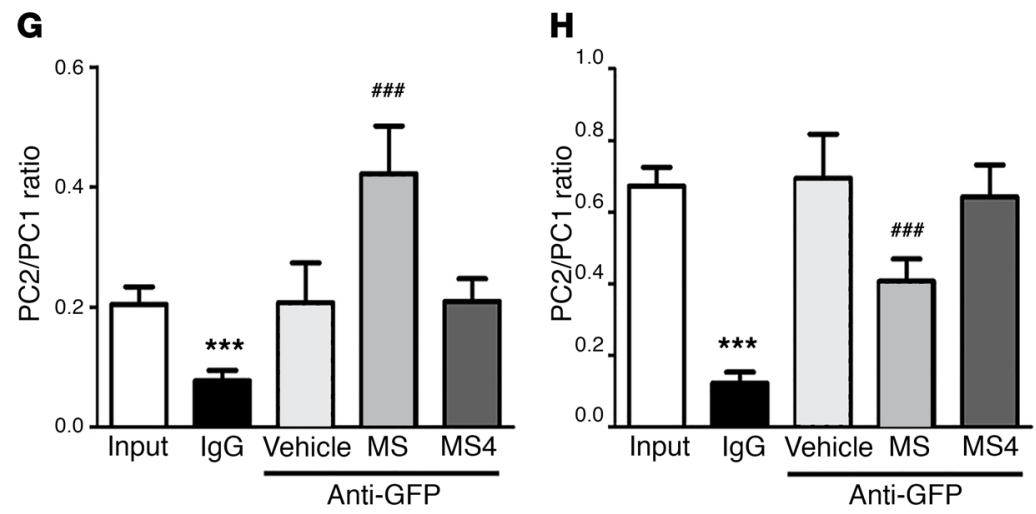
Figure 7. Effects of Zinc01442821 (MS) and its inactive form (MS4) on PC1/PC2 and TAZ complex formation by co-IP assays. (A) Chemical structure of Zinc01442821. (B) Predicted binding mode of Zinc01442821 (ball-and-sticks rendering in CPK colors) in PC2 (light blue). PC1 (red) as bound to PC2 in the crystal structure is superimposed. (C) Co-IP analysis by anti-FLAC for TAZ with either MS analog $(20 \mu \mathrm{M})$ or vehicle (DMSO) control. ( $\mathbf{D}$ and $\mathbf{E}$ ) Quantification of $\mathrm{PC1}$ and $\mathrm{PC2}$ abundance relative to the amount of TAZ. (F) Co-IP analysis by anti-GFP for PC1 with either MS analog $(10 \mu \mathrm{M})$ or vehicle (DMSO) control. (G and $\mathbf{H})$ Quantification of PC2 and TAZ abundance relative to the amount of PC1. Data are presented as the mean \pm SD from 3 independent experiments $(n=3)$. $P$ values were determined by 1-way ANOVA with Newman-Keuls multiple-comparisons test. ${ }^{* *} P<0.001$ compared with input group. ${ }^{\# \# P} P<0.001$ compared with vehicle control group.

MC3T3-E1 osteoblasts in vitro. Zinc01442821 (about 0.5-10 $\mu \mathrm{M}$ ) dose-dependently increased ALPL activity and calcium deposition during osteogenic cultures (Figure 8, C and D). Also, realtime RT-PCR analysis revealed that Zinc01442821 at $10 \mu \mathrm{M}$ concentration markedly stimulated the expression of Runx2 and its downstream gene osteocalcin, but significantly attenuated the expression of Pparg and its downstream gene aP2 (Figure $8 \mathrm{E})$. To determine that $\mathrm{PC} 1$ and $\mathrm{PC} 2$ are mediating the response to Zinc01442821 in osteoblasts, we derived primary osteoblasts from $P k d 1$ - and $P k d 2$-null mice. We found that primary osteoblasts responded to Zinc01442821, but Pkd1- and Pkd2-deficient osteoblasts lost Zinc01442821 stimulation of intracellular calcium (Figure $8 \mathrm{~F}$ ) and TEAD reporter activity (Figure $8 \mathrm{G}$ ).

Finally, it is well known that the acute administration of bone active agents, such as parathyroid hormone, can lead to alterations in osteoblast-related gene expression within hours to days (61). Therefore, we administered Zinc01442821 to WT mice and assessed its effects on the expression of osteoblast and adipocyte markers in bone after 72 hours. Consistent with the in vitro osteoblast culture data, treatment of mice with Zinc01442821 (100 mg/kg) twice daily for 3 days significantly upregulated the expression of Runx2 message and its downstream gene osteocalcin, but inhibited the expression of Pparg message and its downstream gene $a P 2$ (Figure $8 \mathrm{H}$ ). These findings suggest that Zinc01442821 modulates PC1/PC2/TAZ signaling to regulate osteoblastogenesis and adipogenesis both in vitro and in vivo.

In vitro functional assays of Zinc01442821 analogs. We also synthesized 5 close analogs of Zinc01442821 (designated as MS1MS5). MS1 is a methyl ester of Zinc01442821, MS2 is an N-methyl analog of MS1, and MS3, MS4, MS5 have the position of the nitrogen shifted toward the carboxyl moiety of Zinc01442821 (Figure 9A). Using MC3T3-E1 osteoblastic cells, we tested the effects of these analogs on intracellular calcium responses and TAZmediated activation of TEAD reporter activity. We found that the analog MS1 or MS2 significantly increased the peak value of Fluo-4 AM intensity and intracellular calcium levels (Figure 9B) as well as TAZ-mediated TEAD reporter activities (Figure 9C) in MC3T3-E1 osteoblasts, similarly to Zinc01442821. In contrast, MS3, MS4, and MS5 analogs had no effect in these assays (Figure 9C), suggesting that the position of the nitrogen in this scaffold is important for function. Indeed, MS4, unlike Zinc01442821, failed to inhibit PC1/ PC2/TAZ trimeric complex formation (Figure 7, C and D).

\section{Discussion}

In this study, we identify novel interactions between polycystins and TAZ in osteoblasts that create a molecular mechanism whereby the skeleton senses mechanical loading to regulate bone mass. PC1 forms a mechanosensing complex with PC2 (10, 31, 32), and also interacts with TAZ $(33,42)$ to regulate bone mass through the reciprocal control of osteoblastogenesis and adipogenesis. Accordingly, mice with compound deletion of $P k d 1$ and Taz exhibit additive effects to reduce bone mass through reduced osteoblastogenesis and increased bone marrow adipogenesis. An interaction between PC1 and TAZ was demonstrated by co-IP studies. Additional in vitro studies show that PC1-CTT and TAZ translocate to the nucleus to activate Runx2 and inhibit PPAR $\gamma$-dependent gene transcription in response to matrix stiffness and mechanical stretch. Moreover, we discovered a novel small molecule that binds to PC2, enhances TAZ-mediated transcription, and mimics the effects of mechanical loading to stimulate osteoblastogenesis and inhibit adipogenesis in vitro.

Loss-of-function double-heterozygous mice indicate genetic interactions between PC1 and TAZ. Compound-heterozygous $P k d 1^{+/-}$ and $\mathrm{Taz}^{+/-}$mice exhibit additive effects to promote bone loss, reduce osteoblast-mediated bone formation, and increase bone marrow fat. Bone marrow stromal cell cultures derived from compoundheterozygous $\mathrm{Pkd1}^{+-}$and $\mathrm{Taz}^{+-}$mice confirm decreased osteogenesis and increased adipogenesis in vitro. In addition, in primary osteoblast cultures derived from $P k d 1^{\text {null/null }}$, we observed reduced osteoblast markers and increased adipogenic markers. In contrast, primary osteoblast culture from $P k d 2^{\text {null/null }}$ mice exhibited a concordant decrease in both osteoblast and adipocyte gene expression. Ex vivo adenoviral Cre-mediated deletion of $P k d 1$ in primary osteoblasts isolated from $P k d 1^{f / f l}$ mice also resulted in impaired osteoblast differentiation and increased adipogenic markers, whereas adenoviral Cre-mediated deletion of $P k d 2$ resulted in a concordant decrease in both osteogenic and adipogenic markers. These cell culture studies are consistent with our prior finding that conditional deletion of $P k d 1$ in the osteoblast lineage in mice results in inhibition of osteoblastogenesis and stimulation of adipogenesis in vivo, whereas conditional deletion of $P k d 2$ results in concordant inhibition of both osteoblastogenesis and adipogenesis $(30,32)$.

Gain-of-function studies demonstrate that PC1-CTT and TAZ stimulate osteoblast and inhibit adipocyte differentiation, respectively. Transfection of PC1-CTT, TAZ, or Runx2 individually stimulates $O c$ promoter activity, and cotransfection of all 3 constructs exhibits additive effects on osteocalcin gene transcription in C3H10T1/2 cells. Similarly, transfection of PC1-CTT, TAZ, or Pparg individually suppresses $a P 2$ promoter activity, and cotransfection of all 3 constructs results in additive suppression of $a P 2$ gene transcription in C3H1OT1/2 cells. We also found that both hard matrix and mechanical stretch stimulated the polycystins/ TAZ mechanosensing signaling complex in vitro, consistent with the possibility that this complex may integrate the response to different physical forces in the bone microenvironment $(31,33)$. Since these in vitro methods of mechanical loading are nonphysiological, additional studies to explore the response to mechanical loading in compound $P k d 1$ and $T A Z$ heterozygous mutant mouse models are needed to further test our hypothesis in vivo. Furthermore, we found that pharmacological activation of PC1/ 
A

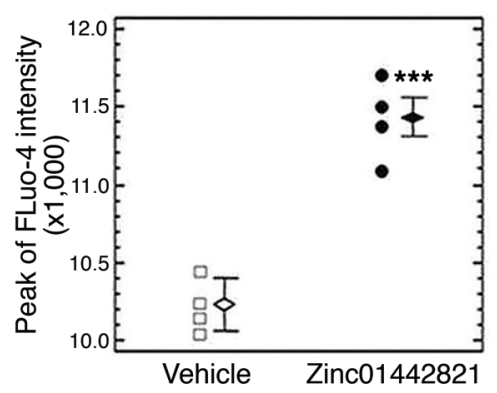

C

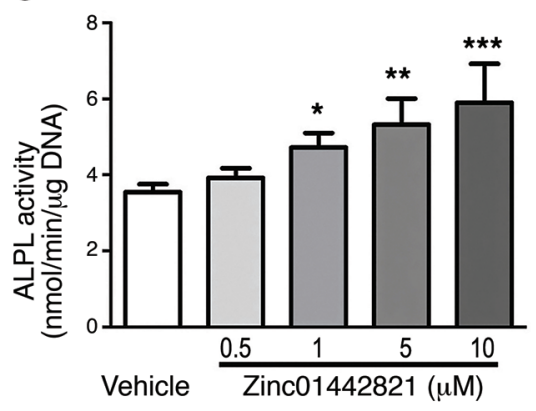

E

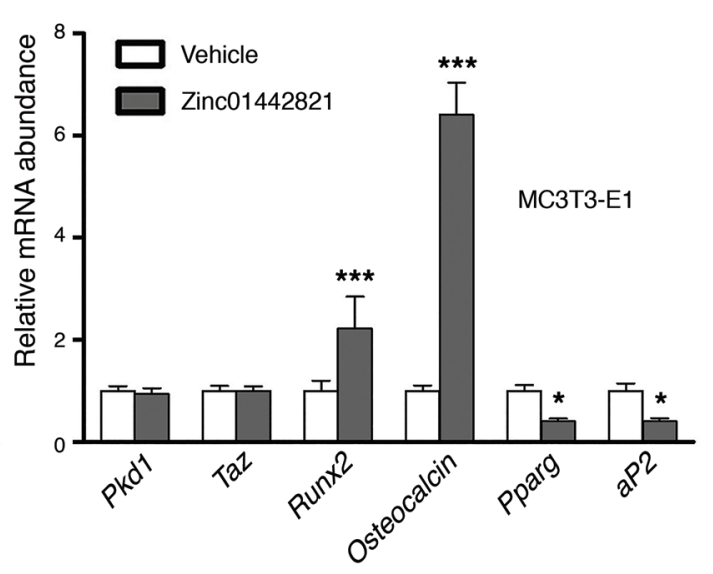

G

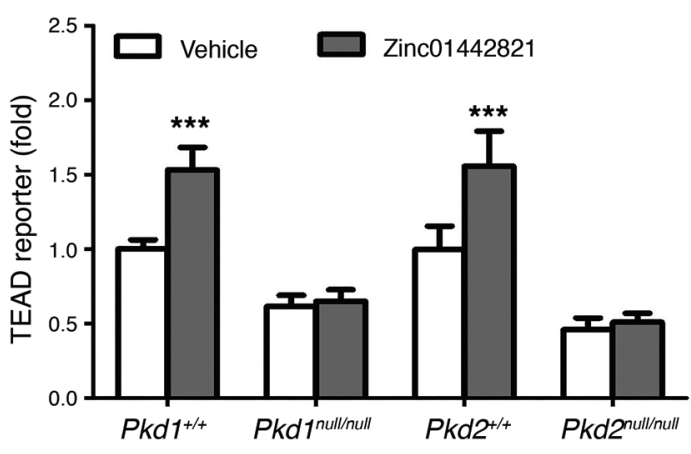

B

D
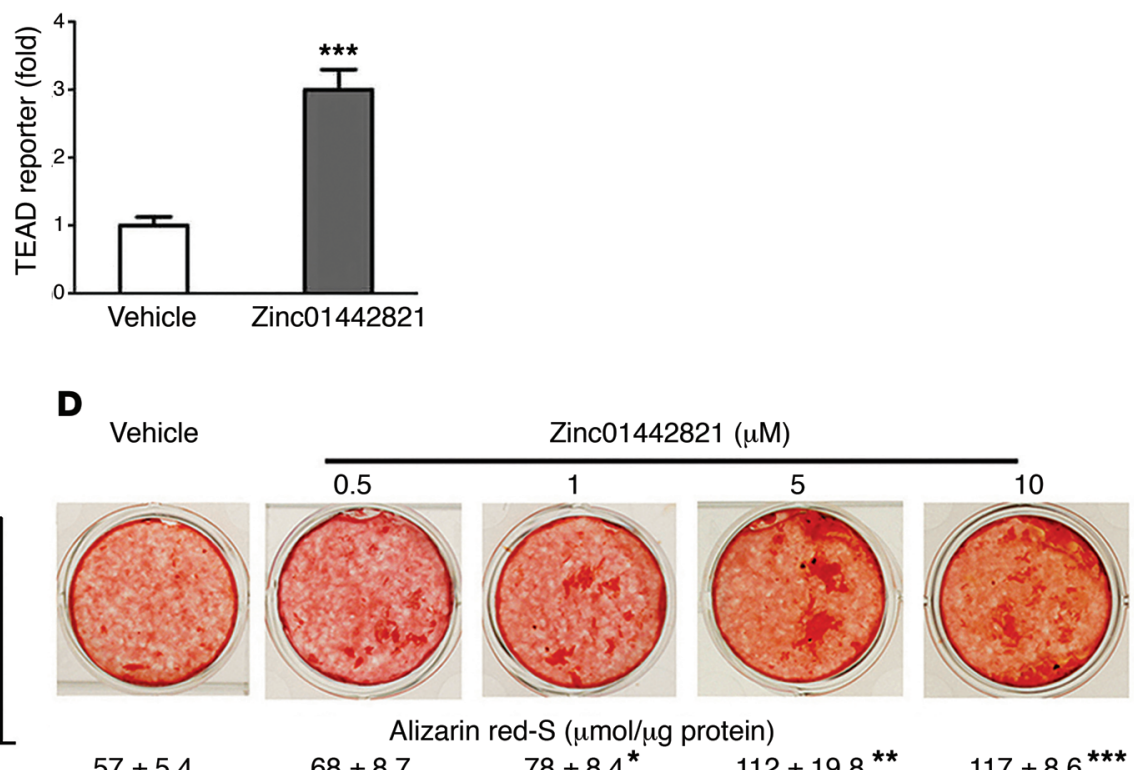

$\mathbf{F}$
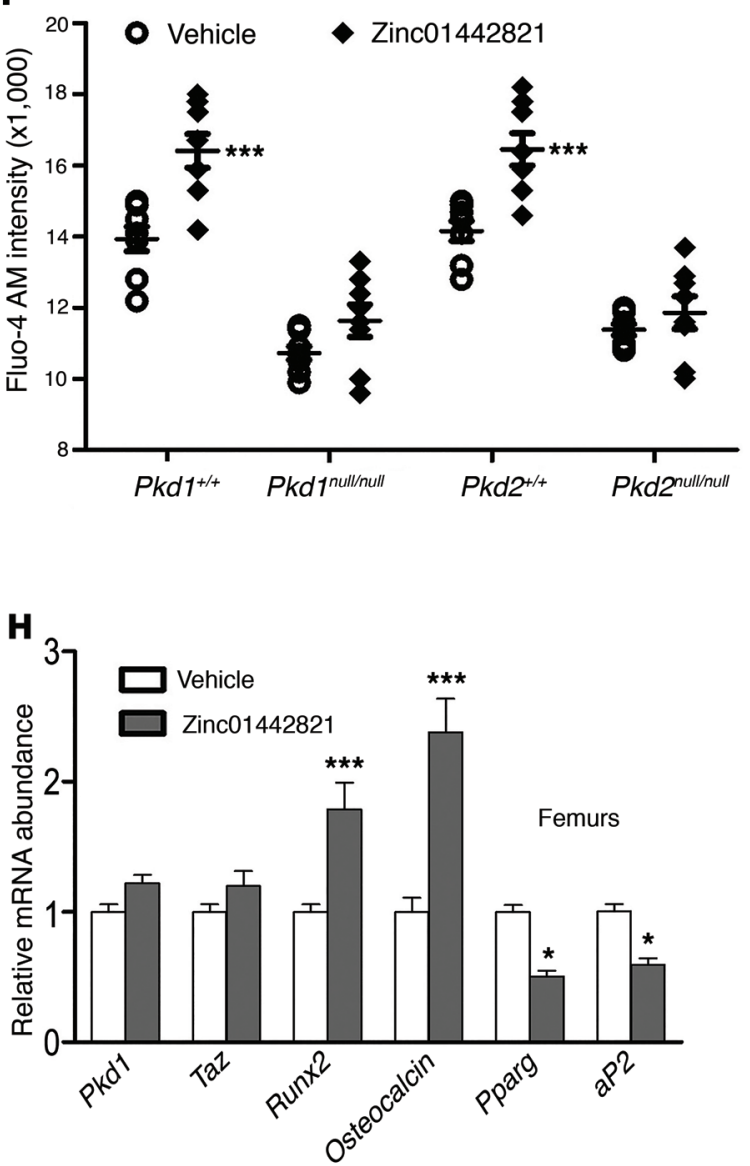
Figure 8. Effects of Zinc01442821 on polycystin/TAZ signaling, osteoblastic differentiation in vitro, and bone-related gene expression in vivo. МСЗТЗ-E1 osteoblasts underwent up to 21 days of culture for osteoblastic differentiation. The [57BL/6] mice were treated with i.p. injection of Zinc01442821 $\left(100 \mathrm{mg} / \mathrm{kg}\right.$ ) or vehicle control twice a day for 3 days. (A) Intracellular [Ca $\left.{ }^{2+}\right]$ response induced by Zinc01442821 (10 $\mu \mathrm{M})$ in MC3T3-E1 osteoblastic cells. (B) Zinc01442821-stimulated $(10 \mu \mathrm{M})$ TAZ-mediated activation of TEAD reporter activities in MC3T3-E1 osteoblastic cells. (C) Dose-dependent effects of Zinc01442821 on ALPL activity at day 15. (D) Dose-dependent effects of Zinc 01442821 on mineralization accumulation by alizarin red $\mathrm{S}$ staining at day 21. (E) Effects of Zinc01442821 $(10 \mu \mathrm{M})$ on the expression of a panel of osteoblast and adipocyte gene markers in MC3T3-E1 cultures at day 21. (F and G) $\left[\mathrm{Ca}^{2+}\right]$, response $(\mathbf{F})$ and TAZ-mediated activation of TEAD reporter activities (G) in WT and polycystin-deficient osteoblasts. (H) Effects of Zinc01442821 on osteogenic and adipogenic markers in femurs from vehicle control- and Zinc01442821-treated mice. Data are expressed as the mean \pm SD from 3 independent experiments $(n=3)$. $P$ values were determined by 1 -way ANOVA with Newman-Keuls multiple-comparisons test or unpaired $t$ test. ${ }^{*} P<0.05$, ${ }^{* *} P<0.01,{ }^{* *} P<0.001$ compared with control group.

PC2/TAZ signaling by Zinc01442821 increased intracellular calcium levels and induced TAZ activation of the TEAD promoter in osteoblasts, in agreement with the effect of extracellular matrix (ECM) stiffness in inducing nuclear translocation of TAZ $(33,62)$. Zinc01442821 also promoted osteoblastogenesis and inhibited adipogenesis in osteoblast cultures. Finally, short-term administration to mice stimulates osteoblastic and inhibits adipocyte message expression in bone in vivo.

Co-IP experiments in both HEK-293T and C3H1OT1/2 cells transfected with $P C 1, P C 2$, and TAZ cDNA constructs show that PC1/PC2 and TAZ form a trimeric complex. PC1-CTT also will pull down TAZ and PC2 in co-IP experiments. In addition, Runx2 and PPAR $\gamma$ are present in the PC1-CTT and TAZ co-IP complexes. The PC1-CTT/TAZ protein complexes translocate from the cytoplasmic compartment to the nucleus of C3H1OT1/2 cells in response to culture on a hard ECM and mechanical stretch. Nuclear translocation of the PC1-CTT/TAZ complex is blocked by treatment with a $\gamma$-secretase inhibitor. The importance of polycystin/ TAZ interactions is further shown by the effects on PC1/PC2/TAZ complex formation of Zinc01442821, a chemical probe developed using structure-based in silico screening $(59,60)$. Interestingly, Zinc01442821, which is predicted to bind to the KRRE cluster of PC2, inhibited trimeric PC1/PC2/TAZ complex formation, but enhanced PC1/PC2 interactions, possibly facilitating TAZ nuclear translocation. TAZ is known to bind to Runx2 and to $\operatorname{PPAR} \gamma$ to respectively enhance and inhibit Runx2 and PPAR $\gamma$ transcriptional activation of target genes (42). ChIP assays were used to assess occupancy of the PC1-CTT/TAZ complexes on targeted gene promoters. Cells cotransfected with PC1-CTT, $T A Z$, and Runx2, as well as cells transfected with PC1-CTT, $T A Z$, and Pparg, identified that complexes formed by these proteins bind to the respective consensus cis-acting sites in the osteocalcin and $a P 2$ promoter $(52,54)$. We previously showed that activation of the PC1/PC2 complex stimulates Runx2 message and protein expression through intracellular calciumdependent nuclear factor I (NFI) pathways in osteoblast $(25,26)$, and the current findings indicate an additional effect of PC1-CTT in enhancing Runx2 effects on targeted genes through a TAZdependent mechanism.
TAZ also binds to PC2, leading to PC2 degradation (41), suggesting that interactions with TAZ may diminish PC2 signaling, and PC1 blocks the PC2/TAZ interaction to prevent TAZmediated degradation of PC2 (41). Thus, the current studies showing that PC1-CTT also binds to TAZ highlight the complexity and competitive nature of the crosstalk between PC1, PC2, and TAZ. Other pathways may also be linked to polycystins. For example, interaction of PC1 with other transcription factors (i.e., NFAT and STAT3) has been described in human osteoblasts that regulate cell proliferation/differentiation via induction of Runx2 $(63,64)$. PC1 is also reported to mediate the response to mechanical strain via potentiation of intracellular calcium and Akt/ $\beta$-catenin pathways in osteoblasts (65).

In conclusion, this study defines a new schema whereby PC1CTT and TAZ form an integrative mechanosensing complex in cells within the osteoblast lineage that differentially regulates osteoblastogenesis and adipogenesis (Figure 10). This schema proposes that mechanical forces, such as ECM stiffness, flow, and mechanical stretch, activate the PC1/PC2 complex (13, 31, 50, 51, 63-66), leading to stimulation of intracellular calcium, induction of PC1-CTT cleavage, and TAZ nuclear translocation to enhance Runx2-mediated induction of osteoblast gene transcription and to inhibit PPAR $\gamma$ and adipogenesis $(33,39,42,44,46,67)$. Together this work identifies a new mechanosensing complex and the feasibility of targeting this complex for treating disorders caused by mechanical unloading and ageing.

\section{Methods}

Mice. All animal research was conducted according to guidelines provided by the NIH and the Institute of Laboratory Animal Resources, National Research Council. The University of Tennessee Health Science Center's Animal Care and Use Committee approved all animal studies (protocol 15-137.0). We obtained the floxed Taz mouse $\left(\operatorname{Taz}^{f / f l}\right)$, which harbors 2 loxP sites flanking exon 2 of the Taz gene, from Jeff Wrana and Helen McNeill (Mount Sinai Hospital and Lunenfeld-Tanenbaum Research Institute, Toronto, Ontario, Canada) (68). We crossed the floxed $T a z^{f / f l}$ mice with $C M V$-Cre mice to generate global TAZ heterozygous mice ( $C M V$-Cre; $\left.\mathrm{Taz}^{+/-}\right)$. These mice were then crossed to WT mice to segregate the $C M V$ Cre transgene from the desired conditional null allele $\left(\mathrm{Taz}^{+/-}\right.$as shown in Figure 1A, equivalent to the Taz null allele). These mice were bred and maintained on a C57BL/6J background for at least 6 generations. The heterozygous $\mathrm{Pkd1}^{+/-}$mice were generated in our laboratory as previously described (46). Then heterozygous $\mathrm{Taz}^{+/-}$ mice were mated with heterozygous $P k d 1^{+-}$mice to generate WT, heterozygous $\mathrm{Pkd1}^{+/-}$, heterozygous $\mathrm{Taz}^{+/}$, and double-heterozygous $\left(P k d 1^{+-} ; \mathrm{Taz}^{+/-}\right)$mice lacking 1 functional allele of both $\mathrm{Pkd1}$ and $\mathrm{Taz}$. These mice were used for phenotypic analysis. The mice were anesthetized with ketamine $(90 \mathrm{mg} / \mathrm{kg})$ and xylazine $(10 \mathrm{mg} / \mathrm{kg})$ for Lunar PIXImus bone densitometer scan, and the mice not useful for experimental purposes were sacrificed by $\mathrm{CO}_{2}$ inhalation plus cervical dislocation. In addition, we used WT C57BL/6J mice at 8 weeks of age to examine the effects of Zinc 01442821 on osteogenesis and adipogenesis in vivo. The mice were treated with i.p. injection of Zinc01442821 (100 mg/kg; Matrix Scientific) or vehicle control (5\% DMSO in PBS solution) twice a day for 3 days. The bone samples were collected 4 hours after the last dose administration. 
<smiles>O=C(O)c1ccccc1-c1ccccc1</smiles>

Zinc01442821 (MS)<smiles>O=C(O)c1ccccc1-c1ccccc1</smiles>

MS1<smiles></smiles>

MS2<smiles>C=CC1=C(c2ccccc2)C=CC1C(=O)O</smiles>

MS3<smiles>C=CC1=C(C(=O)O)C(c2ccccc2)=CC1</smiles>

MS4<smiles>CS(=O)(=O)c1ccccc1-c1ccsc1C(=O)O</smiles>

MS5

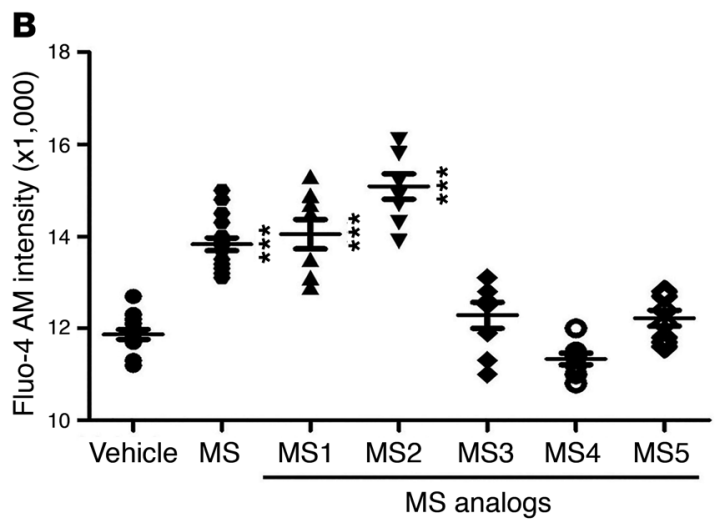

MS analogs

\section{C}

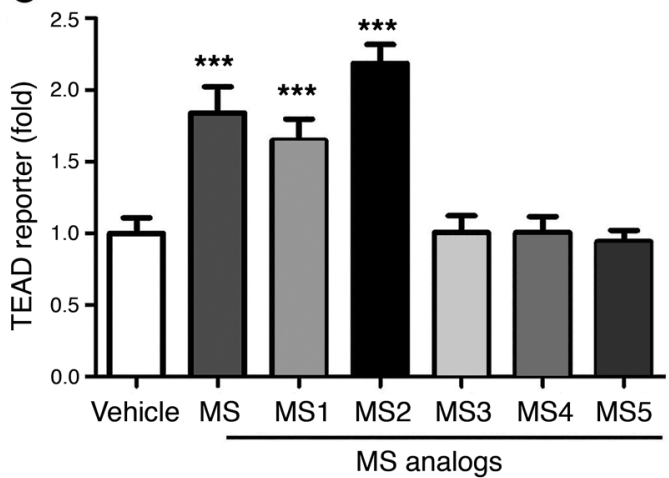

Figure 9. Effects of Zinc01442821 (MS) and its analogs on polycystins/TAZ signaling in osteoblast cultures. (A) Chemical structure of MS and its analogs. (B and $\mathbf{C}$ ) Intracellular $\left[\mathrm{Ca}^{2+}\right]_{i}$ response and TAZ-mediated activation of TEAD reporter activities in MC3T3-E1 osteoblasts with either MS analog (10 $\mu$ M) or vehicle (DMSO) control. Data are expressed as the mean \pm SD $(n=8)$. $P$ values were determined by 1-way ANOVA with Newman-Keuls multiple-comparisons test. ${ }^{* *} P<0.001$ compared with controls.

Bone densitometry, histomorphometric, and micro-CT analysis. BMD of femurs was assessed at 8 weeks of age using a Lunar PIXImus bone densitometer (Lunar Corp.). Calcein (Sigma-Aldrich) double labeling of bone and histomorphometric analyses of periosteal mineral apposition rate in tibiae and osteoclast surface per bone surface (percent) in femurs by TRAP immunostaining were performed using the OsteoMeasure analysis system (OsteoMetrics). The distal femoral metaphyses were also scanned using a Scanco $\mu$ CT 40 (Scanco Medical AG). A 3D image analysis was done to determine bone volume and cortical thickness as previously described (30-32).

$\mathrm{OsO}_{4}$ staining and micro-CT analysis. Whole intact femurs and tibiae with encapsulated marrow were dissected from 8-week-old mice, fixed for 48 hours in phosphate-buffered paraformaldehyde, and decalcified in $14 \%$ EDTA for 2 weeks. Then the femurs and tibiae were stained for 2 hours in $2 \%$ aqueous osmium tetroxide $\left(\mathrm{OsO}_{4}\right)$. The bones were rinsed in water for 48 hours and then scanned at 6 $\mu \mathrm{m}$ resolution using a Scanco $\mu \mathrm{CT} 40,45 \mathrm{kVp}$ and $177 \mu \mathrm{A}$. Quantification of fat volume, density, and distribution throughout the marrow was registered to low-contrast decalcified bone as our laboratory previously described $(30,32)$.

BMSC cultures. BMSCs from 8-week-old mice were prepared as previously described (69). Briefly, the femurs and tibiae were dissected, the ends of the bones were cut, and the marrow was flushed out with 2 $\mathrm{ml}$ of ice-cold $\alpha$-minimum essential medium ( $\alpha$-MEM) containing $10 \%$ (vol/vol) FBS using a 22-gauge needle and syringe. Cells were seeded into 12-well plates or 60-mm plates at a density of $1 \times 10^{7}$ cells $/ \mathrm{ml}$ and cultured for 3 days in $\alpha$-MEM supplemented with $15 \%$ vol/vol FBS, $1 \%$ penicillin/streptomycin $(\mathrm{P} / \mathrm{S})$, and kept in a humidified incubator with $5 \% \mathrm{CO}_{2}$ and $95 \%$ air at a temperature of $37^{\circ} \mathrm{C}$. On day 3 , all nonadherent cells were removed with the first medium change, and then the adherent cells (representing BMSCs) received the subsequent treatments. For osteoblastic differentiation, the adherent cells were grown in osteogenic medium ( $\alpha$-MEM containing $10 \% \mathrm{vol} / \mathrm{vol}$ FBS supplemented with $5 \mathrm{mM}$ $\beta$-glycerophosphate and $25 \mu \mathrm{g} / \mathrm{ml}$ of ascorbic acid) for periods of up to 12 days. ALPL activity and alizarin red S histochemical staining for mineralization were performed as previously described (69). For quantification of mineralization, alizarin red $S$ was extracted with $10 \%$ cetylpyridinium chloride and assessed at $562 \mathrm{~nm}$. Total DNA content was measured with a double-strand DNA quantitation reagent and kit (Molecular Probes). Protein concentrations of the supernatant were determined with a total protein assay kit (Bio-Rad). We also used TRIzol reagent to isolate the total RNAs for real-time RT-PCR analysis as previously described (46).

Osteoblastic cell cultures. Immortalized $P k d 1^{\text {null } / \text { null }}, P k d 2^{\text {null } / \text { null }}$, and MC3T3-E1 osteoblastic cell lines (ATCC) were cultured in $\alpha$-MEM containing $10 \% \mathrm{FBS}$ and $1 \% \mathrm{P} / \mathrm{S}$ as previously described $(31,32)$. For intracellular calcium measurements, Fluo-4 or Fluo-4 AM (Invitrogen) was used according to the manufacturer's instructions (25). For TAZ signaling study, the immortalized $P k d 1^{\text {null/null }}$ and $P k d 2^{\text {null/null }}$ cells were cultured in the osteogenic differentiation media for 48 hours. Then the cells were lysed with $150 \mu \mathrm{l}$ of T-PER with $1 \times$ Halt protease inhibitor and $1 \mathrm{mM}$ PMSF per well. After 30 -second sonication 3 times, total cell lysates were centrifuged at $13,000 \mathrm{~g}$ for 10 minutes, and supernatants were stored at $-80^{\circ} \mathrm{C}$ until use for the following Western blot analysis.

Mechanical cell stretch. C3H10T1/2 (ATCC) cells were plated onto 6-well plates with Collagen Type I surface (Flexcell International) for 24 hours and then subjected to a 6-hour stretch regimen by software-controlled vacuum applied to a loading station housed in a humidified $5 \% \mathrm{CO}_{2}$ incubator at $37^{\circ} \mathrm{C}$ (Flexcell FX-4000T, Flex- 


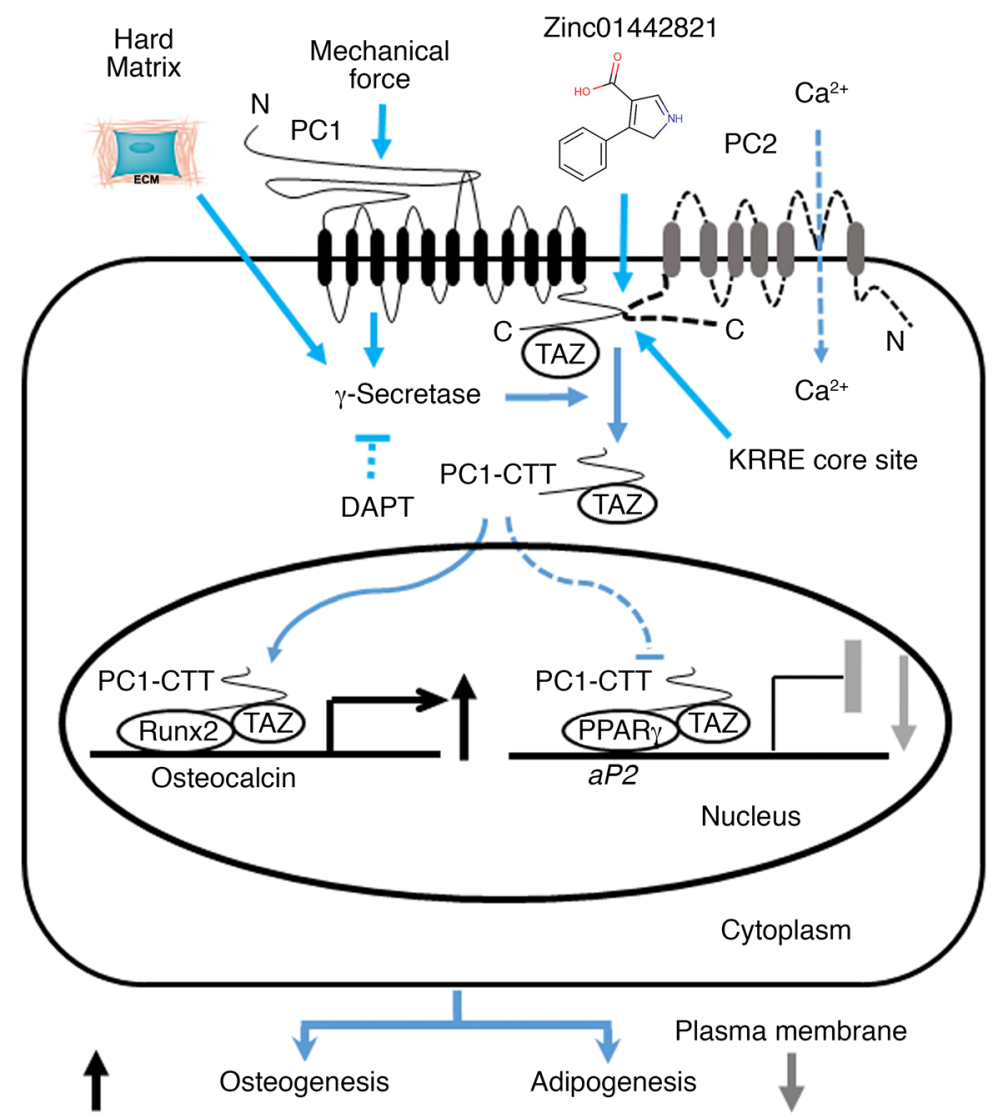

Figure 10. Schematic of PC1 and TAZ interaction in regulation of osteogenesis and adipogenesis in bone marrow. Mechanical force and hard matrix regulate Runx2mediated $O c$ and PPAR $\gamma$-mediated $a P 2$ promoter activities through PC1-CTT and TAZ nuclear translocation in mesenchymal stem cells.

cell International). Each cycle consisted of 0.5 seconds of stretch $(10 \%)$ and 0.5 seconds of relaxation for a total of 15 cycles per minute, which achieves the maximum effect on osteogenic gene expression in BMSCs and C3H10T1/2 cells as previously described for osteogenesis (55-57). The replicate control plates consisted of cells grown on the same flexible surface but not subjected to mechanical stretch. We immediately collected cell samples for the measurements of $\gamma$-secretase activity, cleavage of PC1-CTT, and PC1-CTT and TAZ nuclear translocation at a completion of 6 hours of stretch. The cells were processed for luciferase activity assays after 18 hours when subjected to 6 hours of stretch (70).

$\gamma$-Secretase assay. Cellular $\gamma$-secretase activities were quantified using an assay based on a previous report (71). Briefly, C3H1OT1/2 cells were harvested in cell lysis buffer A containing $20 \mathrm{mM}$ HEPES (pH 7.0), $150 \mathrm{mM} \mathrm{KCl,} 2 \mathrm{mM}$ EDTA, 1\% [(3-cholamidopropyl) dimethylammonio]-2-hydroxy-1-propanesulfonate(CHAPSO;wt/vol; Sigma-Aldrich), and Protease Inhibitor Cocktail (Thermo Fisher Scientific). Lysates were centrifuged at 9,300 $\mathrm{g}$ for 1 minute at $4^{\circ} \mathrm{C}$ to remove nuclei and large cell debris. Supernatants were collected, and the protein concentration was quantified. Fifty micrograms of protein was added to an opaque 96-well plate (Nunclon; Nunc) and made to a final volume of $200 \mu \mathrm{l}$ with assay buffer containing $50 \mathrm{mM}$ Tris$\mathrm{HCl}$ (pH 6.8), 2 mM EDTA, and 0.25\% CHAPSO (wt/vol); $10 \mathrm{mM}$ of fluorogenic amyloid $\beta$-peptide precursor protein-derived probe acting as a $\gamma$-secretase substrate (Sigma-Aldrich) was added to the plate. Controls used were the peptide probe and cell lysates alone. Samples were incubated at $37^{\circ} \mathrm{C}$ for 18 hours in the dark. Fluorescence was measured using a Synergy H4 Hybrid Reader (BioTek Instruments Inc.), with excitation wavelength at $340 \mathrm{~nm}$ and emission wavelength at $460 \mathrm{~nm}$. Background fluorescence of the peptide probe was subtracted from all readings.

Computational small-molecule discovery. Zinc01442821 (4-phenyl-1H-pyrrole-3-carboxylic acid, $\mathrm{C}_{11} \mathrm{H}_{9} \mathrm{NO}_{2}$ ) was identified by a computationally driven, structure-based, and virtual docking screening, following approaches previously described $(59,60)$. Briefly, we used the PC1:PC2 coiled-coil model of Zhu et al. (17) to perform a docking screening of 1,000 diverse fragment-like molecules from the ZINC database using the MOE docking facility (Chemical Computing Group) version 2015, in an induced-fit setting. Zinc01442821 ranks among the top molecules predicted to interact with residues in the binding pocket of PC2 in which PC1 binds. This compound was selected based on the number of similar compounds and potential chemical modifications that can be identified in the ZINC database for follow-up screening.

To induce osteoblastic differentiation, the MC3T3-E1 cells were plated at a density of $2 \times 10^{4}$ cells per well in a 12-well plate and $4 \times 10^{4}$ cells per well in a 6-well plate and grown up to 21 days in osteogenic medium in the presence of Zinc01442821 $(0,0.5,1$, and $10 \mu \mathrm{M})$. ALPL activity and alizarin red S histochemical staining for mineralization were performed as previously described $(30,32)$. Total DNA content was measured with a PicoGreen dsDNA quantitation reagent and kit (Molecular Probes). Protein concentrations of the supernatant were determined with a Bio-Rad protein assay kit.

Real-time quantitative RT-PCR and Western blot analysis. For realtime quantitative RT-PCR, $1.0 \mu \mathrm{g}$ total RNA isolated from either the long bone of 6-week-old mice or BMSCs cultured for 8 days in differentiation media was reverse transcribed as previously described $(30,32)$. PCR reactions contained $20 \mathrm{ng}$ template (cDNA), $375 \mathrm{nM}$ each forward and reverse primers, and $1 \times$ EvaGreen Supermix (Bio-Rad) in $10 \mu \mathrm{l}$. The threshold cycle (Ct) of tested-gene product from the indicated genotype was normalized to the $\mathrm{Ct}$ for cyclophilin $\mathrm{A}$. Then the tested-gene product versus cyclophilin A was normalized to the mean ratio of WT or control group, which had been set to 1 .

For Western blot analysis, protein concentrations of the supernatant were determined with a total protein assay kit (Bio-Rad). Equal quantities of protein were subjected to $4 \%-12 \%$ Bis-Tris or $3 \%-8 \%$ Tris-Acetate gradient gels (Invitrogen) and were analyzed with standard Western blot protocols as previously described (30, 32). Polycystin-1 antibody (7E12, sc-130554), polycystin-1 antibody (C-20, sc-10372), polycystin-2 antibody (H-280, sc-25749), and polycystin-2 antibody (YCE2, sc-47734) were purchased from Santa Cruz Biotechnology. Purified mouse TAZ antibody (no. 560236) was purchased from BD Biosciences. Phosphorylated TAZ (Ser 89, sc-17610) and $\beta$-actin (sc-47778) antibodies were from Santa Cruz Biotechnology. The intensity of bands was quantified using ImageJ software (NIH; http://rsb.info.nih.gov/ij/).

Transient transfection, promoter reporter activity, and co-IP. C3H10T1/2 cells were cultured in minimum essential medium (MEM) 
containing $10 \%$ FBS and $1 \% \mathrm{P} / \mathrm{S}$. To examine the effect of PC1-CTT and TAZ interaction on Runx 2 or PPAR $\gamma$ activity, $5 \times 10^{6}$ C3H1OT1/2 cells were transfected with $1.0 \mu \mathrm{g}$ of PC1-CTT, $1.0 \mu \mathrm{g}$ of TAZ, and/or $1.0 \mu \mathrm{g}$ of Runx 2 or Pparg expression constructs in combination with 3.0 $\mu \mathrm{g}$ of mouse osteocalcin or $a P 2$ promoter luciferase reporter and $0.6 \mu \mathrm{g}$ of Renilla luciferase-null as internal control plasmid by electroporation using a Cell Line optimal transfection kit according to the manufacturer's protocol (Amaxa Inc.). A total of $6.6 \mu \mathrm{g}$ of plasmid DNAs was used for each electroporation. The transfected cells were plated in 12-well plates and harvested 32 hours after transfection. Cells were lysed in $1 \times$ reporter lysis buffer, a luciferase assay was performed using a dual luciferase assay kit (Promega), and activity was measured with a Synergy H4 Hybrid Reader. To test the effect of PC1 C-tail cleavage on Oc or $a P 2$ reporter activity, the transfected cells were treated with vehicle (DMSO) control or $50 \mu \mathrm{M}$ DAPT (Sigma-Aldrich; a $\gamma$-secretase inhibitor) for the last 12 hours and harvested 36 hours after transfection.

For co-IP analyses, $5 \times 10^{6}$ HEK-293T cells were transfected with either 3.0 $\mu$ g of FLAG-tagged TAZ, $3.0 \mu \mathrm{g}$ of GFP-tagged full-length PC1, and $3.0 \mu$ g of myc-tagged full-length $P C 2$ (Baltimore PKD Core Center) or $3.0 \mu \mathrm{g}$ of FLAG-tagged TAZ, $3.0 \mu \mathrm{g}$ of myc-tagged PC1-CTT, and $3.0 \mu \mathrm{g}$ of Runx 2 or Pparg expression constructs for 48 hours. The transfected cells were washed once with ice-cold $1 \times$ PBS and added to appropriate ice-cold $1 \times$ IP lysis buffer (Pierce Biotechnology) containing $1 \times$ protease inhibitors. The cellular lysates were centrifuged at approximately $13,000 \mathrm{~g}$ for $10 \mathrm{~min}-$ utes to pellet the cell debris at $4^{\circ} \mathrm{C}$, and the supernatants were collected to a new tube for protein concentration determination. Fifty microliters of either anti-FLAG, anti-myc, or anti-GFP magnetic beads was added into $400 \mu \mathrm{l}$ of the lysate supernatant as suggested in the manufacturer's protocol (Sigma-Aldrich). The mixtures were incubated with gentle agitation for 2 hours or overnight at $4^{\circ} \mathrm{C}$. The magnetic beads were washed 3 times with $1 \mathrm{ml}$ of cold lysis buffer and suspended in $30 \mu \mathrm{l}$ of Laemmli's sample buffer. The supernatant samples were boiled for 3-5 minutes and collected for further analysis. Ten microliters of the sample per lane was loaded in either $3 \%-8 \%$ Tris-Acetate or $4 \%-12 \%$ Bis-Tris gradient gels for Western blot analysis. Anti-PPAR $\gamma(\mathrm{C} 26 \mathrm{H} 12,2435)$ and anti-YAP/TAZ (D24E4, 8418) were purchased from Cell Signaling Technology. AntiRunx2 mAb was purchased from MBL International (D130-3). FLAG Epitope Tag (DYKDDDDK) Antibody (FG4R, MA1-91878) was purchased from Pierce Biotechnology.

Quantitative ChIP. The quantitative ChIP analyses were performed using an enzymatic chromatic IP kit from Cell Signaling Technology according to the manufacturer's instructions with the following modifications (72). Briefly, $5 \times 10^{6} \mathrm{C} 3 \mathrm{H} 10 \mathrm{~T} 1 / 2$ cells were transfected with $3.0 \mu \mathrm{g}$ of FLAG-tagged TAZ, $3.0 \mu \mathrm{g}$ of myc-tagged PC1-CTT, and 3.0 $\mu \mathrm{g}$ of Runx2 or Pparg expression constructs for 48 hours and treated with $1 \%$ formaldehyde to cross-link chromatin. Immunoprecipitations were carried out with $2 \mu \mathrm{g}$ of antibodies (anti-FLAG from SigmaAldrich, anti-myc from Pierce Biotechnology, anti-Runx2 and antiPPAR $\gamma$ from Cell Signaling Technology). The specific protein/DNA complex was reversely cross-linked, and DNA fragments were purified. For $O c$ promoter, a real-time PCR amplification was performed using primers (forward 5'-CCAGCTGAGGCTGAGAGAGA-3', reverse 5'-CTAATTGGGGGTCATGTGCT-3') located in the OSE2 site (a known Runx2-binding site) as previously reported (72). Primers (forward 5'-CAGTATGGCTTGAAGACCGC-3', reverse 5'-AGAGAGAGAGGACAGGGAGG-3') amplifying exon 4, which does not contain any Runx2-binding sites, were used as a control sequence. For $a P 2$ promoter, a DNA amplification was performed using primers (forward 5'-AGCAGGAATCAGGTAGCTGG-3', reverse 5'-GTTACAAGGCAAGGAAGGGC- $3^{\prime}$ ) located around the ARE6 site (a known PPAR $\gamma$-binding site) as previously reported (54). Primers (forward 5'-GTGCAGAAGTGGGATGGAAA-3', reverse 5'-CATTCCACCACCAGCTTGTC-3') amplifying exon 3 , which does not contain any PPAR $\gamma$-binding sites, were used as a control sequence. Fold enrichment reflects the ratio of OSE2 or ARE6/ control sequence in the IP versus the input samples. In addition, histone $\mathrm{H} 3$ rabbit $\mathrm{mAb}(2 \mu \mathrm{g})$ and normal rabbit $\operatorname{IgG}(1 \mu \mathrm{g})$ were used as positive or negative controls, respectively.

Acrylamide hydrogels and cytoplasmic and nuclear protein extraction. To assess the effects of ECM stiffness on PC1-CTT and TAZ nuclear translocation, we created various ECM stiffness using acrylamide hydrogel methods, as previously described (33). The C3H1OT1/2 cells were plated in acrylamide hydrogel-coated hard $(40 \mathrm{kPa})$ and soft $(0.5$ $\mathrm{kPa}$ ) matrix under growth conditions for 72 hours. For Western blot analysis, cytoplasmic and nuclear extracts from cultured C3H10T1/2 cells were prepared using a NE-PER nuclear extraction kit (Pierce Chemical). Protein concentrations were determined with a Bio-Rad protein assay kit. Equal quantities of protein were subjected to NuPAGE 4\%-12\% Bis-Tris Gel (Invitrogen) and analyzed with standard Western blot protocol. Anti-YAP/TAZ (D24E4, 8418) was purchased from Cell Signaling Technology. FLAG Epitope Tag (DYKDDDDK) Antibody (FG4R, MA1-91878) was purchased from Pierce Biotechnology. Lamin A/C antibody was purchased from Santa Cruz Biotechnology. Signals were detected using HRP-conjugated secondary antibodies (Santa Cruz Biotechnology) and an enhanced chemiluminescence detection kit (ECL Plus Western Blotting Detection Reagents, GE Healthcare).

Synthesis of MS analogs MS1 to MS5. Five close MS analogs, MS1 to MS5, were synthesized to provide preliminary structure-activity relationship information based on the computation model shown Figure 7B. All compounds have a purity of greater than $98 \%$, and their structures were authenticated by standard analytical chemistry analyses.

Statistics. We evaluated differences between 2 groups by unpaired $t$ test, and between multiple groups by 1-way ANOVA with NewmanKeuls multiple-comparisons test. All values are expressed as means \pm SD. All computations were performed using commercial biostatistics software (GraphPad Software Inc.).

\section{Author contributions}

ZX, JB, CRY, and LDQ wrote the manuscript. ZX, LC, JH, and $\mathrm{BD}$ performed the in vitro and in vivo experimental studies. JB designed and performed the computational studies. CRY and JB analyzed the computational results and identified compounds of interest, HC and WL designed and synthesized MS analogs MS1 to MS5, and WL, CMW, JCS, and LDQ guided the research and reviewed and edited the manuscript.

\section{Acknowledgments}

This work was supported by NIH grants R01-DK083303, R01AR045955, and R01-AR37308 to LDQ and HL123540 and HL131526 to CMW.

Address correspondence to: L. Darryl Quarles, Coleman College of Medicine Building, Suite B216, University of Tennessee Health Science Center, 956 Court Avenue, Memphis, Tennessee 38163, USA. Phone: 901.448.1459; E-mail: dquarles@uthsc.edu. 
1. Sen B, Xie Z, Case N, Ma M, Rubin C, Rubin $\mathrm{J}$. Mechanical strain inhibits adipogenesis in mesenchymal stem cells by stimulating a durable $\beta$-catenin signal. Endocrinology. 2008;149(12):6065-6075.

2. Zayzafoon M, Gathings WE, McDonald JM. Modeled microgravity inhibits osteogenic differentiation of human mesenchymal stem cells and increases adipogenesis. Endocrinology. 2004;145(5):2421-2432.

3. Justesen J, Stenderup K, Ebbesen EN, Mosekilde L, Steiniche T, Kassem M. Adipocyte tissue volume in bone marrow is increased with aging and in patients with osteoporosis. Biogerontology. 2001;2(3):165-171.

4. Moerman EJ, Teng K, Lipschitz DA, LeckaCzernik B. Aging activates adipogenic and suppresses osteogenic programs in mesenchymal marrow stroma/stem cells: the role of PPAR- $\gamma 2$ transcription factor and TGF- $\beta / \mathrm{BMP}$ signaling pathways. Aging Cell. 2004;3(6):379-389.

5. David V, et al. Mechanical loading down-regulates peroxisome proliferator-activated receptor $\gamma$ in bone marrow stromal cells and favors osteoblastogenesis at the expense of adipogenesis. Endocrinology. 2007;148(5):2553-2562.

6. Rubin CT, et al. Adipogenesis is inhibited by brief, daily exposure to high-frequency, extremely low-magnitude mechanical signals. Proc Natl Acad Sci U S A. 2007;104(45):17879-17884.

7. Du L, Fan H, Miao H, Zhao G, Hou Y. Extremely low frequency magnetic fields inhibit adipogenesis of human mesenchymal stem cells. Bioelectromagnetics. 2014;35(7):519-530.

8. Chan CK, et al. Identification and specification of the mouse skeletal stem cell. Cell. 2015;160(1-2):285-298.

9. Takada I, Kouzmenko AP, Kato S. Wnt and PPAR $\gamma$ signaling in osteoblastogenesis and adipogenesis. Nat Rev Rheumatol. 2009;5(8):442-447.

10. Xiao Z, Quarles LD. Physiological mechanisms and therapeutic potential of bone mechanosensing. Rev Endocr Metab Disord. 2015;16(2):115-129.

11. Araç D, et al. A novel evolutionarily conserved domain of cell-adhesion GPCRs mediates autoproteolysis. EMBO J. 2012;31(6):1364-1378.

12. Forman JR, Qamar S, Paci E, Sandford RN, Clarke J. The remarkable mechanical strength of polycystin-1 supports a direct role in mechanotransduction. J Mol Biol. 2005;349(4):861-871.

13. Nauli SM, et al. Polycystins 1 and 2 mediate mechanosensation in the primary cilium of kidney cells. Nat Genet. 2003;33(2):129-137.

14. Qian F, Germino FJ, Cai Y, Zhang X, Somlo S, Germino GG. PKD1 interacts with PKD2 through a probable coiled-coil domain. Nat Genet. 1997;16(2):179-183.

15. Hanaoka K, et al. Co-assembly of polycystin-1 and -2 produces unique cation-permeable currents. Nature. 2000;408(6815):990-994.

16. Tsiokas L, Kim E, Arnould T, Sukhatme VP, Walz G. Homo- and heterodimeric interactions between the gene products of PKD1 and PKD2. Proc Natl Acad Sci U S A. 1997;94(13):6965-6970.

17. Zhu J, et al. Structural model of the TRPP2/ PKD1 C-terminal coiled-coil complex produced by a combined computational and experimental approach. Proc Natl Acad Sci U S A.
2011;108(25):10133-10138.

18. Piontek K, Menezes LF, Garcia-Gonzalez MA, Huso DL, Germino GG. A critical developmental switch defines the kinetics of kidney cyst formation after loss of Pkd1. Nat Med. 2007;13(12):1490-1495.

19. Kim I, et al. Conditional mutation of $\mathrm{Pkd} 2$ causes cystogenesis and upregulates beta-catenin. J Am Soc Nephrol. 2009;20(12):2556-2569.

20. Qian F, Watnick TJ, Onuchic LF, Germino GG. The molecular basis of focal cyst formation in human autosomal dominant polycystic kidney disease type I. Cell. 1996;87(6):979-987.

21. Koptides M, Mean R, Demetriou K, Pierides A, Deltas CC. Genetic evidence for a transheterozygous model for cystogenesis in autosomal dominant polycystic kidney disease. Hum Mol Genet. 2000;9(3):447-452.

22. Chauvet V, et al. Expression of PKD1 and PKD2 transcripts and proteins in human embryo and during normal kidney development. Am J Pathol. 2002;160(3):973-983.

23. Boulter C, Mulroy S, Webb S, Fleming S, Brindle K, Sandford R. Cardiovascular, skeletal, and renal defects in mice with a targeted disruption of the Pkd1 gene. Proc Natl Acad Sci U S A. 2001;98(21):12174-12179.

24. Watnick TJ, et al. Somatic mutation in individual liver cysts supports a two-hit model of cystogenesis in autosomal dominant polycystic kidney disease. Mol Cell. 1998;2(2):247-251.

25. Xiao Z, Zhang S, Magenheimer BS, Luo J, Quarles LD. Polycystin-1 regulates skeletogenesis through stimulation of the osteoblast-specific transcription factor RUNX2-II. J Biol Chem. 2008;283(18):12624-12634.

26. Xiao Z, et al. Cilia-like structures and polycystin-1 in osteoblasts/osteocytes and associated abnormalities in skeletogenesis and Runx2 expression. J Biol Chem. 2006;281(41):30884-30895.

27. Khonsari RH, et al. Multiple postnatal craniofacial anomalies are characterized by conditional loss of polycystic kidney disease 2 ( $\mathrm{Pkd} 2$ ). Hum Mol Genet. 2013;22(9):1873-1885.

28. Mesner LD, et al. Bicc1 is a genetic determinant of osteoblastogenesis and bone mineral density. J Clin Invest. 2014;124(6):2736-2749.

29. Lu W, et al. Comparison of Pkd1-targeted mutants reveals that loss of polycystin- 1 causes cystogenesis and bone defects. Hum Mol Genet. 2001;10(21):2385-2396.

30. Xiao Z, Zhang S, Cao L, Qiu N, David V, Quarles LD. Conditional disruption of Pkd1 in osteoblasts results in osteopenia due to direct impairment of bone formation. J Biol Chem. 2010;285(2):1177-1187.

31. Xiao Z, et al. Conditional deletion of Pkd1 in osteocytes disrupts skeletal mechanosensing in mice. FASEB J. 2011;25(7):2418-2432.

32. Xiao Z, et al. Osteoblast-specific deletion of Pkd2 leads to low-turnover osteopenia and reduced bone marrow adiposity. PLoS One. 2014;9(12):e114198.

33. Dupont $\mathrm{S}$, et al. Role of YAP/TAZ in mechanotransduction. Nature. 2011;474(7350):179-183.

34. Varelas X. The Hippo pathway effectors TAZ and YAP in development, homeostasis and disease. Development. 2014;141(8):1614-1626.
35. Aragona M, et al. A mechanical checkpoint controls multicellular growth through YAP/ TAZ regulation by actin-processing factors. Cell. 2013;154(5):1047-1059.

36. Makita R, et al. Multiple renal cysts, urinary concentration defects, and pulmonary emphysematous changes in mice lacking TAZ. Am J Physiol Renal Physiol. 2008;294(3):F542-F553.

37. Hossain Z, et al. Glomerulocystic kidney disease in mice with a targeted inactivation of Wwtr1. Proc Natl Acad Sci U S A. 2007;104(5):1631-1636.

38. Merrick D, Bertuccio CA, Chapin HC, Lal M, Chauvet V, Caplan MJ. Polycystin-1 cleavage and the regulation of transcriptional pathways. Pediatr Nephrol. 2014;29(4):505-511.

39. Merrick D, et al. The $\gamma$-secretase cleavage product of polycystin-1 regulates TCF and CHOP-mediated transcriptional activation through a p300-dependent mechanism. Dev Cell. 2012;22(1):197-210.

40. Merrick DM, Wu J, Baggs JE, Hogenesch JB, Caplan MJ. Polycystin-1 stimulates skeletogenesis via TAZ-mediated activation of RunX2. FASEB J. 2012;26(1 suppl):lb811.

41. Tian Y, et al. TAZ promotes PC2 degradation through a SCFbeta-Trcp E3 ligase complex. Mol Cell Biol. 2007;27(18):6383-6395.

42. Hong JH, et al. TAZ, a transcriptional modulator of mesenchymal stem cell differentiation. Science. 2005;309(5737):1074-1078.

43. Cui CB, Cooper LF, Yang X, Karsenty G, Aukhil I. Transcriptional coactivation of bone-specific transcription factor Cbfa1 by TAZ. Mol Cell Biol. 2003;23(3):1004-1013.

44. Jung $\mathrm{H}$, et al. Augmentation of PPARgamma-TAZ interaction contributes to the anti-adipogenic activity of KR62980. Biochem Pharmacol. 2009;78(10):1323-1329.

45. Yang JY, et al. Osteoblast-targeted overexpression of TAZ increases bone mass in vivo. PLoS One. 2013;8(2):e56585.

46. Qiu N, Cao L, David V, Quarles LD, Xiao Z. Kif3a deficiency reverses the skeletal abnormalities in Pkd1 deficient mice by restoring the balance between osteogenesis and adipogenesis. PLoS One. 2010;5(12):e15240.

47. Kanai F, et al. TAZ: a novel transcriptional co-activator regulated by interactions with 14-3-3 and PDZ domain proteins. ЕMBO J. 2000;19(24):6778-6791.

48. Bertuccio CA, et al. Polycystin-1 C-terminal cleavage is modulated by polycystin-2 expression. J Biol Chem. 2009;284(31):21011-21026.

49. Bertuccio CA, Caplan MJ. Polycystin-1C terminus cleavage and its relation with polycystin-2, two proteins involved in polycystic kidney disease. Medicina (B Aires). 2013;73(2):155-162.

50. Low SH, et al. Polycystin-1, STAT6, and P100 function in a pathway that transduces ciliary mechanosensation and is activated in polycystic kidney disease. Dev Cell. 2006;10(1):57-69.

51. Chauvet V, et al. Mechanical stimuli induce cleavage and nuclear translocation of the polycystin-1 C terminus. J Clin Invest. 2004;114(10):1433-1443.

52. Ducy P, Karsenty G. Two distinct osteoblastspecific cis-acting elements control expression of a mouse osteocalcin gene. Mol Cell Biol. 1995;15(4):1858-1869. 
53. Ducy P, Zhang R, Geoffroy V, Ridall AL, Karsenty G. Osf2/Cbfa1: a transcriptional activator of osteoblast differentiation. Cell. 1997;89(5):747-754.

54. Graves RA, Tontonoz P, Spiegelman BM. Analysis of a tissue-specific enhancer: ARF6 regulates adipogenic gene expression. Mol Cell Biol. 1992;12(3):1202-1208.

55. Wang C, et al. Mechanical stimulation promote the osteogenic differentiation of bone marrow stromal cells through epigenetic regulation of Sonic Hedgehog. Exp Cell Res. 2017;352(2):346-356.

56 . Gao J, et al. Cyclic stretch promotes osteogenesisrelated gene expression in osteoblast-like cells through a cofilin-associated mechanism. Mol Med Rep. 2016;14(1):218-224.

57. Wu Y, Zhang X, Zhang P, Fang B, Jiang L. Intermittent traction stretch promotes the osteoblastic differentiation of bone mesenchymal stem cells by the ERK1/2-activated Cbfa1 pathway. Connect Tissue Res. 2012;53(6):451-459.

58. Li R, et al. Mechanical stretch inhibits mesenchymal stem cell adipogenic differentiation through TGF $\beta 1 /$ Smad2 signaling. J Biomech. 2015;48(13):3665-3671.

59. Evangelista W, et al. Ensemble-based docking: from hit discovery to metabolism and toxicity predictions. Bioorg Med Chem. 2016;24(20):4928-4935.
60. Xiao Z, et al. A computationally identified compound antagonizes excess FGF-23 signaling in renal tubules and a mouse model of hypophosphatemia. Sci Signal. 2016;9(455):ra113.

61. Osagie-Clouard L, Sanghani A, Coathup M, Briggs T, Bostrom M, Blunn G. Parathyroid hormone 1-34 and skeletal anabolic action: the use of parathyroid hormone in bone formation. Bone Joint Res. 2017;6(1):14-21.

62. Azzolin L, et al. Role of TAZ as mediator of Wnt signaling. Cell. 2012;151(7):1443-1456.

63. Dalagiorgou G, et al. Mechanosensor polycystin-1 potentiates differentiation of human osteoblastic cells by upregulating Runx2 expression via induction of JAK2/STAT3 signaling axis. Cell Mol Life Sci. 2017;74(5):921-936.

64. Dalagiorgou G, Piperi C, Georgopoulou U, Adamopoulos C, Basdra EK, Papavassiliou AG. Mechanical stimulation of polycystin-1 induces human osteoblastic gene expression via potentiation of the calcineurin/NFAT signaling axis. Cell Mol Life Sci. 2013;70(1):167-180.

65. Wang H, Sun W, Ma J, Pan Y, Wang L, Zhang W. Polycystin-1 mediates mechanical strain-induced osteoblastic mechanoresponses via potentiation of intracellular calcium and Akt/ $\beta$-catenin pathway. PLoS One. 2014;9(3):e91730.
66. Gordon WR, et al. Mechanical allostery: evidence for a force requirement in the proteolytic activation of Notch. Dev Cell. 2015;33(6):729-736.

67. Talbot JJ, et al. The cleaved cytoplasmic tail of polycystin-1 regulates Src-dependent STAT3 activation. J Am Soc Nephrol. 2014;25(8):1737-1748.

68. Reginensi A, et al. Yap- and Cdc42-dependent nephrogenesis and morphogenesis during mouse kidney development. PLoS Genet. 2013;9(3):e1003380.

69. Xiao ZS, et al. Effect of asymmetric dimethylarginine on osteoblastic differentiation. Kidney Int. 2001;60(5):1699-1704.

70. Speight P, Kofler M, Szaszi K, Kapus A. Contextdependent switch in chemo/mechanotransduction via multilevel crosstalk among cytoskeleton-regulated MRTF and TAZ and TGF $\beta$-regulated Smad3. Nat Commun. 2016;7:11642.

71. Farmery MR, Tjernberg LO, Pursglove SE, Bergman A, Winblad B, Näslund J. Partial purification and characterization of gamma-secretase from post-mortem human brain. J Biol Chem. 2003;278(27):24277-24284.

72. Zhang S, Xiao Z, Luo J, He N, Mahlios J, Quarles LD. Dose-dependent effects of Runx2 on bone development. J Bone Miner Res. 2009;24(11):1889-1904. 